\title{
Semantic relations underlie non-linear planning in complex noun phrases
}

\author{
Jens Roeser, Mark Torrance, Thom Baguley
}

Department of Psychology, Nottingham Trent University, Nottingham, UK

\section{Author Note}

Jens Roeser (D) https://orcid.org/0000-0002-4463-0923

Correspondence concerning this article should be addressed to Jens Roeser, Department of Psychology, Nottingham Trent University, 50 Shakespeare St, Nottingham NG1 4FQ. E-mail: jens.roeser@ntu.ac.uk 


\begin{abstract}
When producing a noun phrase whether or not pre-planning extends beyond the initial noun varies with the phrase's syntactic structure. However it is not clear on what basis - conceptual or syntactic - the production system determines that pre-planning is necessary. In two experiments $\left(N_{\mathrm{s}}=32,64\right)$ participants produced noun phrases in response to picture arrays. Surface form was held constant but scope of the initial determiner was manipulated by varying the contrastive functions of the first and the second noun (e.g., The man with the painting [but not the girl with the painting] vs. The man with the painting [but not the man with the ball]). Evidence from eye movement data revealed a stronger tendency for early planning in the extended-scope condition. This is evidence that pre-planning requirements of structurally complex noun phrase are determined prior to the processing of syntax and lexis.

Keywords: Semantic structure; planning scope; language production; hierarchical planning; sentence processing; eye tracking
\end{abstract}




\section{Semantic relations underlie non-linear planning in complex noun phrases}

\section{Introduction}

Intuitively, reading, listening, speaking and writing are all processes that unfold over the linear order of the surface structure of the language that is being processed. The underlying structure of language, however, is hierarchically complex: Semantic and syntactic dependencies exist between words that are adjacent and also between words that are non-adjacent. In a simple phrase such as the blue hat the determiner "the" is semantically and syntactically dependent on "hat" and not "blue". Structural dependencies affect how language is processed. In reading, increasing distance between co-dependent elements increases processing difficulty (e.g. Gibson, 2000; Lewis \& Vasishth, 2005). In speech the modifier "blue" in the blue hat needs to be pre-planned before production-onset (i.e. in advance of starting articulation) in languages in which the adjective comes before the nominal head but not in languages in which the adjective follows the noun (Brown-Schmidt \& Konopka, 2008; Schriefers \& Teruel, 1999; Schriefers, Teruel \& Meinshausen, 1998).

In order to start articulation speakers need to pre-plan some linguistic unit. This unit may or may not be structurally complex. In the language production model outlined by Bock and Ferreira (2014) syntactic dependencies are build from rudimentary abstract semantic representations ("scaffolds") which feed into syntactic assembly. The process that translates these semantic scaffolds into syntactic and lexical representations is generally referred to as "grammatical encoding" (Bock \& Levelt, 1994; Costa \& Caramazza, 2002; Dell \& O’Seaghdha, 1992; V. S. Ferreira \& Slevc, 2007; Garrett, 1975; Levelt, 1989). Advance grammatical encoding appears, in some contexts, to scope over just the initial noun (e.g. Brown-Schmidt \& Konopka, 2008; Brown-Schmidt \& Tanenhaus, 2006; F. Ferreira \& Swets, 2002; Griffin, 2001; Griffin \& Bock, 2000; Zhao \& Yang, 2016). Importantly, however, due to circumstances that are not yet well understood, pre-planning can also scope across complex syntactic units (Allum \& Wheeldon, 2007, 2009; 
Brown-Schmidt \& Konopka, 2015; F. Ferreira, 1991; Hardy, Segaert \& Wheeldon, 2019, 2020; Konopka, 2012; Kuchinsky, 2009; Kuchinsky, Bock \& Irwin, 2011; E.-K. Lee, Brown-Schmidt \& Watson, 2013; Levelt \& Maasen, 1981; Martin, Crowther, Knight, Tamborello II \& Yang, 2010; Martin, Yan \& Schnur, 2014; Nottbusch, 2010; Nottbusch, Weingarten \& Sahel, 2007; Roeser, Torrance \& Baguley, 2019; Smith \& Wheeldon, 1999; Swets, Jacovina \& Gerrig, 2014; Wagner, Jescheniak \& Schriefers, 2010; Wheeldon, 2011, 2012; Wheeldon, Ohlson, Ashby \& Gator, 2013; Wheeldon, Smith \& Apperly, 2011; Zhao, Alario \& Yang, 2015; Zhao \& Yang, 2013). The evidence comes from increased attention to stimulus items associated with later sentence elements or increased stimulus-to-sentence onset durations when co- or subordinated nouns were added to the sentence-initial subject phrase - e.g. The $A$ and the $B$ compared to The $A$ - which seems to be independent of planning costs associated with lexical retrieval (e.g., retrieval of the B; Griffin, 2001, Hardy, Segaert \& Wheeldon, 2018, Roeser, 2017, Roeser et al., 2019, Smith \& Wheeldon, 1999, Wheeldon et al., 2013, Zhao \& Yang, 2016).

Recent research on sentence production has moved away from attempting to determine a universal planning scope - how far must planning stretch into the surface form of the target sentences before production start - to explore the particular conditions under which pre-planning must extend beyond the initial element (typically a determiner-noun pair; Bock \& Ferreira, 2014, Konopka \& Brown-Schmidt, 2014, Konopka \& Kuchinsky, 2015, Konopka \& Meyer, 2014, Kuchinsky, 2009, Kuchinsky et al., 2011, E.-K. Lee et al., 2013, Momma, Slevc \& Phillips, 2015). So for target utterance $A-B$ - $C$, in some syntactic, semantic or lexical contexts articulation can start as soon as $A$ has been planned. In other contexts $A$ - $B$ must be planned. In still others planning must go beyond $A$ to involve activation of both $A$ and $C$, but not not necessarily $B$ (i.e. planning scope does not necessarily follow the linear sequence of the surface form of the target utterance). This raises two questions: What are the particular conditions under which planning needs to scope over both $A$ and $C$ rather than just $A$ ? And how does the language production 
system decide whether or not this more extensive pre-planning is required? The latter question is the focus of the research that we present in this paper.

The possibility that planning might deviate from the linear structure of the target utterance was recognised by Konopka and Meyer (2014). They argued that there are two routes by which the intended message can be converted into a linguistic structure: a non-relational route that gives precedence to the individual elements of the message and a relational route that prioritises the relationships among these elements. Some authors attribute advance planning primarily to non-relational information (Brown-Schmidt \& Konopka, 2008, 2015; Gleitman, January, Nappa \& Trueswell, 2007), others to the relationships among message elements (Bock, Irwin \& Davidson, 2004; Kuchinsky, 2009). Konopka and Meyer (2014) suggested that speakers can prioritise either process depending on ease of access. The authors tested this in a priming study and found that recently-experienced structures and lexical material determines the extend to which the verb guides advance planning. Similarly Kuchinsky (2009) reported that when linear processing is not easily available planning unfolds in a non-linear order; this was found if the name of the first noun, but not the name of the verb, was difficult to retrieve (see also Van de Velde, Meyer \& Konopka, 2014). Several other studies have found evidence of relational, non-linear planning at the clause-level (e.g. Bock \& Griffin, 2000; Bunger, Papafragou \& Trueswell, 2013; Do, 2018; Do \& Kaiser, 2019; Konopka \& Bock, 2009; Konopka \& Brown-Schmidt, 2014; Konopka \& Kuchinsky, 2015; Meyer, 1996, 1997; Momma \& Ferreira, 2019; Momma et al., 2015; Van de Velde et al., 2014). All of these studies attribute the need for non-linear planning to the activation of the verb and its arguments stressing the verb's fundamental role for encoding of the sentence's syntactic organisation in existing models of language production (Antón-Méndez, 2020; Bock \& Ferreira, 2014; Bock \& Levelt, 1994; Chang, Dell \& Bock, 2006; F. Ferreira, 2000; Kempen \& Hoenkamp, 1987; Levelt, 1989).

Pre-planning of complex subject noun phrases might involve a similar relational 
process that maps semantics onto syntax. As discussed above, coordinated subject noun phrases are known to involve pre-planning beyond the sentence-initial noun. One explanation for the increased planning demands is that the order of coordinated noun phrases is syntactically and semantically arbitrary - the meaning of the $A$ and the $B$ is identical to the $B$ and the $A$ (Allum \& Wheeldon, 2007). Creating a linear order, therefore, adds to planning demands. For phrases with subordinated modifiers (e.g. The $A$ above the $B$ ), the evidence is conflicting. These might either require less (Allum \& Wheeldon, 2007; Zhao \& Yang, 2013) or more (Nottbusch, 2010; Nottbusch et al., 2007) advance planning than coordinated noun phrases. This might be because head nouns and subordinated nouns constitute different functional domains (Allum \& Wheeldon, 2007; Zhao \& Yang, 2013) or because of the structural relationship between a head noun and its modifier (Nottbusch, 2010; Nottbusch et al., 2007), respectively. Additionally there may be effects due to the semantic integration of the head and the subordinated noun (Solomon \& Pearlmutter, 2004).

E.-K. Lee et al. (2013) directly investigated whether advance planning of complex noun phrases is mediated by syntactic dependencies between phrase elements. In response to arrays of images participants generated utterances in the form of instructions to the researcher such as (Click on) the fork of the king that is below the apple. The image location of either the fork or the king in relation to the image of an apple changed the underlying syntactic structure of the utterance without affecting its surface form. Low attachment of the relative clause conveyed that the king is below the apple; high attachment expressed that the fork was below the apple. The authors found longer onset latencies for high-attaching phrases, and argued that this was due to the need for advance planning of the relative clause. This finding suggests that advance planning of noun phrases is mediated by syntactic dependencies between phrase elements. There might indeed be some processing linked to the organisation of the noun phrase that determines whether or not advance planning has to extend beyond the first noun. In particular it 
could be that some or all of the dependencies within the noun phrase must be pre-planned.

Non-adjacent dependencies as in the E.-K. Lee et al. (2013) example suggest pre-planning of structure beyond the initial determiner-noun pair: Even though there is pressure in the production system for linear planning, output must be delayed if the to-be-produced noun phrase contains dependencies. Arguably, however, whether or not the production system is required to pre-plan these syntactic dependencies cannot in itself be based on establishing those dependencies: The production system's decisions to start output or to delay until further syntactic units are planned cannot rely on planning of those units. We therefore hypothesise that whether or not syntactic planning beyond the initial determiner-noun pair is obligated is established at a pre-syntactic level. Instead, it seems likely that the feasibility of incremental assembly (versus the need to advance-plan) relies on a semantic analysis of the target message.

Semantic-relational information is clearly relevant to deciding whether or not to pre-plan a coordinate or subordinated noun phrase. If the relationship between two nouns does not affect advance planning, it would be difficult to explain the E.-K. Lee et al. (2013) finding and why sentence-initial noun phrases such as The $A$ and the $B$ systematically lead to longer planning durations than simple (The A) (e.g. Martin et al., 2014; Wagner et al., 2010; Wheeldon et al., 2013) and, in some instances, subordinated noun phrases (The A above the B) (Allum \& Wheeldon, 2007, 2009). Several authors have proposed that the extent to which complex subject noun phrases require pre-planning is determined during grammatical encoding (see Griffin, 2003, for an alternative proposal). For example the linearisation of an initially unordered message plan involves the generation of more complex syntactic frames (Smith \& Wheeldon, 1999; Wheeldon et al., 2013) or pre-planning of thematic elements beyond the first noun (Allum \& Wheeldon, 2007, 2009). Both explanations entail some form of abstract semantic scaffolding that (a) decides whether the noun order is arbitrary or structured (e.g. subordinated), and (b) determines the semantic relation of the nouns that form conjoined noun phrases (e.g. The $A$ and the 
$B$ ) different from disjunctions (e.g. The $A$ or the $B$ ). The processor needs to create an abstract representation of the semantic relationship between message elements before the language production system can initiate grammatical encoding. The processor's decision about whether non-linear planning is necessary or whether linear planning is permitted, is therefore the key to determining whether to plan syntactic assembly before or after production onset.

The hypothesis that we explore is the experiments that we report in this paper is, therefore, that linearity in advance planning of complex noun phrases is determined at the semantic level. Specifically, we determined whether relational semantic information induces planning beyond the first determiner-noun pair in subordinated noun phrases when the surface structure is held constant. We report two image-description experiments in which we manipulated referential contrast. Referential contrast (often referred to as focus) distinguishes between current information and potential alternatives (Jackendoff, 1972). In spoken English contrast is typically marked by prosodic prominence (Selkirk, 1995). The shelf above the WINDOW (capitals indicate prosodic prominence) refers to a shelf that is located above a window rather than a shelf above an alternative reference object, say, a door. In this example the semantic scope of the determine (The) embraces both nouns. On the other hand The SHELF above the window distinguishes between two or more different objects located above a window: The semantic scope embraces just the first noun. This semantic property was used to examine the influence of semantic relations on phrase pre-planning without varying the syntactic structure and lexical content.

To test whether the semantic scope affects advance planning, we recorded both eye movements on the stimulus array and response-onset latency. We used an image description task to establish whether semantic contrast increases the planning scope. If semantic relations determine the need for advance planning of the surface form then target phrases where the determiner scopes over both nouns (i.e. the contrastive function is served by the referent of the second noun) will be associated with greater planning scope 
(is less likely to be planned incrementally) than target phrases where the scope is narrow (i.e. the contrastive function is served by the referent of the first noun).

Eye movement on the stimulus array provides an effective indicator of rapid processes such as conceptual planning (Griffin \& Bock, 2000; Swets et al., 2014). Data from eye tracking are necessary in the context of our experiments to understand what information provided by the visual array was used and when it became relevant (see e.g. Barr, 2008; Meyer \& Lethaus, 2004; Mirman, Dixon \& Magnuson, 2008). Several similar studies have reported effects on eye movements but no effect on response latency (e.g. Gleitman et al., 2007; Griffin \& Bock, 2000; Konopka \& Meyer, 2014; Swets et al., 2014). For example, E.-K. Lee et al. (2013), in the study discussed above, failed in a follow-up experiment to replicate the effect of attachment on response latency. In our study eye movements allowed us to establish whether attention was shifted to the critical referent before production onset (Experiment 1) and when attention was shifted from one referent in the target utterance to the next (Experiment 2; see e.g. Griffin, 2004, Griffin \& Bock, 2000, Konopka \& Meyer, 2014, Meyer \& Lethaus, 2004).

In Experiment 1, and in one of two conditions in Experiment 2 participants typed their responses. This was for two reasons. First, there is a pressure for fluency in spoken production that is not present in writing. In speech, hesitation has communicational effect. Writers, however, can pause where and for as long as they like. It is possible, therefore, that previous studies with spoken output wrongly interpreted effects of the (modality specific) requirement that spoken output is fluent as evidence for fundamental (modality independent) pre-planning requirements (but see Roeser et al., 2019). A second reason for exploring these effects in written production relates to the need to keep output form identical in narrow and wide scope conditions. Semantic contrast in English is typically encoded by means of prosodic prominence (e.g. the shelf above the WINDOW) and therefore affects the surface structure of the produced utterance. The addition of prosodic stress in speech might therefore present a potential confound in the present study. For 
these reasons we evaluated effects primarily in the written modality. However, as we report, effects did in fact replicate in speech.

\section{Experiment 1}

Scope was manipulated for the first and the second noun in the output string. It is known that pre-planning takes into account the structural function of the phrase-initial noun (Brown-Schmidt \& Konopka, 2015 but see Allum \& Wheeldon, 2007). Therefore, we elicited pre- (possessive) and postnominal (e.g. prepositional) modifier phrases as the pre-syntactic nature of semantic planning should induce advance planning across noun phrase modification type. This modifier manipulation allows us to account for alternative

explanations related to head position (Allum \& Wheeldon, 2007) and stimulus array that, otherwise, would be orthogonal to the Scope manipulation. Head-initial phrases may allow planning of the modifier to be postponed until after production onset. A phrase-initial modifier, by contrast, may require pre-planning across the head-noun. Our hypothesis is that a contrastive second noun requires pre-planning as it is relevant for the referential meaning of the noun phrase. Crucially, the surface form - the syntactic structure and the lexical content - were held constant.

\section{Method}

\section{Participants}

Thirty-two psychology students $(27$ female, 5 male, mean age $=19.7$ years, $S D=3.0$, range: 18-32) participated as part of a research-reward scheme. All participants were self-reported native speakers of British English, free of psycholinguistic impairments, and had normal or corrected-to-normal vision. Eight participants were replaced because they failed to produce descriptions that matched the targeted structures. 


\section{Design}

Participants were asked to write, by typing on a keyboard, phrases that unambiguously identified the coloured object in an array of images such as that shown in Figure 1. We manipulated determiner scope in two different phrase structures: prenominal modifiers (e.g. The cowboy's hamburger is green, in which the noun hamburger is modified by the preceding cowboy's) or a noun with a postnominal modifier (e.g. The cowboy (that is) above the hamburger is green, in which cowboy is modified by the following above the hamburger). Participants were asked to use prenominals when a line connected the target and another image (see Figure 1a and 1b) or to use a postnominal phrase, indicating the vertical location of the coloured image, when this line was absent (see Figure 1d and 1c). We hypothesised that pre-planning would be affected by determiner scope in both prenominal and postnominal conditions.

Scope - the manipulation that tested our hypothesis - was manipulated by varying the contrast required to unambiguously identify the coloured image. For example, in the target phrase The cowboy's hamburger is green the determiner The scopes over just cowboy when there is a choice of possessor (cowboy or robot) - the narrow scope condition - and but includes the (non adjacent) hamburger in its scope when there is a choice of possessed object (hamburger or hairbrush).

We recorded eye movements within the stimulus array. All images were areas of interest (AOI), but the critical AOI was the image representing the second noun in the target phrase (hamburger in The cowboy's hamburger is green). The size of the AOI corresponded to the size of the image. We calculated the proportion of eye samples on this image prior to production onset. We also recorded onset latency, timed from appearance of the stimulus array on the computer screen to the first keystroke of the written output. 

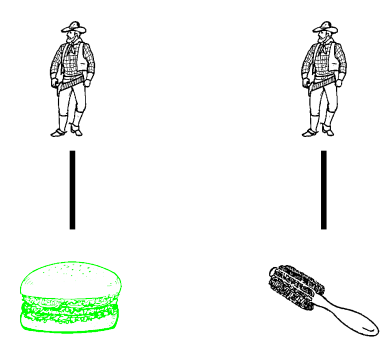

(a) Prenominal, wide scope: The cowboy's hamburger is green [not the cowboy's brush]
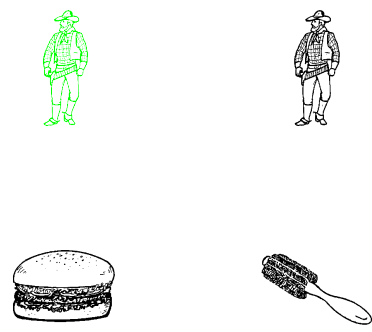

(c) Postnominal, wide scope: The cowboy above the hamburger is green [not the cowboy above the brush]

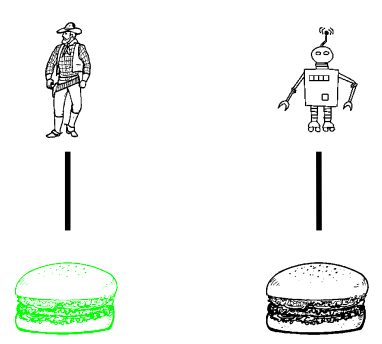

(b) Prenominal, narrow scope: The cowboy's hamburger is green [not the robot's hamburger]
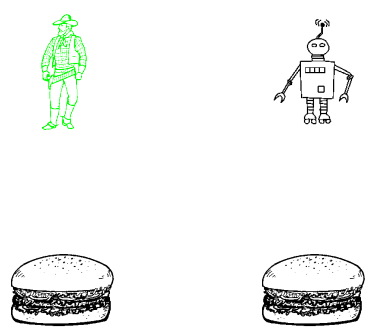

(d) Postnominal, narrow scope: The cowboy above the hamburger is green [not the robot above the hamburger]

\section{Figure 1}

Example stimulus arrays (Experiment 1).

\section{Materials}

Thirty-two items with arrays of four images were created with animate objects in one row and inanimate objects in the other row. Black and white drawings were taken from the database of the International Picture Naming Project (E. Bates et al., 2003; Székely et al., 2003; Székely et al., 2005; Székely et al., 2004). Items were distributed across four Latin square lists to counterbalance images across Phrase Type (prenominal, postnominal) and Scope (wide, narrow). Also, the horizontal and vertical positions of the target group were counterbalanced to prevent predictability of the position of the target (i.e. of the coloured image). The colour of the target was varied between items (green, red, blue, 
yellow). A list of the stimulus materials can be found in Appendix A.

48 fillers were added to each list. Filler arrays contained fewer than three images with no coloured image, or one or more coloured images. Each list was presented in random order.

\section{Apparatus}

Eye movements were recorded using an SR Research EyeLink 1000. The eye tracker was desk mounted and used in remote mode to ensure free head movements. Eye data were sampled monocular (right eye) on a frequency of $500 \mathrm{~Hz}$. The participant was seated 55 to $60 \mathrm{~cm}$ away from the lens. The experiment was built in SR Research Experiment Builder. Stimuli were displayed on a 19" ViewSonic Graphic Series (G90fB) CRT monitor with a screen resolution of 1,280 × 1,024 pixels and $85 \mathrm{~Hz}$ refresh rate on using an Intel Core $2 \mathrm{PC}$.

\section{Procedure}

Trials started with a centred filled circle $(21 \times 21$ pixels $)$ that required a fixation of $200 \mathrm{~ms}$ to trigger display of the image array. The images, size $200 \times 200$ pixels (including transparent margins) appeared equally spaced around the centre of the screen. A text box $(896 \times 50$ pixels $)$ was provided at the bottom of the screen in which the produced text was displayed. Participants were instructed to position their fingers over the keyboard at the start of the trial and to respond quickly and accurately as soon as possible after appearance of the stimulus array. All images were shown on the screen until the participant finished typing their description. When the participant had finished typing their response they pressed return to proceed to the next trial. They were told that they could pause between trials if they wished. The entire experiment lasted approximately 45 minutes.

Experimental sessions started with a 9-point eye-tracker calibration and validation. Participants were familiarised with the intended constructions and then completed 10 practice items. The experimenter monitored descriptions during practice and corrected the participant where necessary. Eye-tracking was recalibrated before the start of the 
experimental trials, and during the experiment just if the fixation trigger failed.

\section{Results}

Data were screened prior to analysis. Sentences that did not match the required structures were excluded from the analysis (2.64\%). Trials with onset latencies $>14$ secs $(0.4 \%)$ and sentence production durations $>30$ secs $(1.17 \%)$ were discarded. Trials where output was disfluent - where participants paused for $>2.5$ secs or where they rephrased their text during production - were removed (12.99\%). In total, $15.4 \%$ of the data were removed. For the analysis of eye data further $0.7 \%$ trials were removed because more than $75 \%$ of eye samples before production onset were outside of the AOIs.

Statistical inference used Bayesian linear mixed effects models (Gelman et al., 2014; Kruschke, 2014; McElreath, 2016) implemented in the probabilistic programming language Stan and the R interfaces Rstan (Carpenter et al., 2016; Hoffman \& Gelman, 2014; Stan Development Team, 2015a, 2015b) and the rstanarm package (Goodrich, Gabry, Ali \& Brilleman, 2020). Models were fitted with weak, locally uniform priors (see McElreath, 2016; Nicenboim \& Vasishth, 2016; Sorensen, Hohenstein \& Vasishth, 2016) and run with 2,000 iterations on 4 chains with a warm-up of 1,000 iterations and no thinning. Model convergence was confirmed by the Rubin-Gelman statistic $(\hat{R}=1)$ (Gelman \& Rubin, 1992) and inspection of the Markov chain Monte Carlo chains.

All models were fitted with maximal random effects structure, i.e. random intercepts for participants and items and by-participant and by-item slope adjustments for all predictors (Baayen, Davidson \& Bates, 2008; Barr, Levy, Scheepers \& Tily, 2013; D. Bates, Kliegl, Vasishth \& Baayen, 2015). Predictors were sum coded and the $95 \%$ posterior probability mass - 95\% probability interval (henceforth, $95 \% \mathrm{PI}^{1}$ ) - was

\footnotetext{
${ }^{1}$ Probability intervals are sometimes referred to as credible intervals. Unlike confidence intervals they have a straightforward understanding as the range within which the true parameter value is likely to occur with, in the present case, 95\% probability (Morey, Hoekstra, Rouder, Lee \& Wagenmakers, 2016).
} 
calculated from the posterior samples.

To assess the strength of support for the effects of interest over the null hypothesis, Bayes Factors (henceforth, BF) were calculated using the Savage-Dickey method (see Dickey, Lientz et al., 1970; Nicenboim \& Vasishth, 2016; Wagenmakers, Lodewyckx, Kuriyal \& Grasman, 2010). BF $=2$, for example, indicates that the alternative hypothesis is twice as likely compared to the null hypothesis given the data. BFs larger than 5 indicate moderate and larger than 10 strong evidence for a statistically meaningful effect (see e.g. Baguley, 2012; Jeffreys, 1961; M. D. Lee \& Wagenmakers, 2014).

R-scripts and data are available at: doi.org/10.6084/m9.figshare.5435923.v1

\section{Eye data}

We calculated the proportion of eye samples on the object that was the referent for the second noun (N2 - e.g. the picture of the hamburger in Figure 1) that occurred during the period prior to production onset. For statistical analysis the proportion of eye samples was converted to empirical logits (see Barr, 2008; Mirman et al., 2008). A Bayesian linear mixed effects model was fitted with main effects for Modifier position and Scope, and their interaction. To account for the multimodal distribution of the data, the models were specified with three mixture components, a combination of three normal distributions, varying the location of each intercept and its variance parameter.

Inferred parameter values from this model can be found in Figure 2. There was strong evidence supporting a Scope effect for prenominals, with a higher proportion of samples on N2 when N2 was contrastive (i.e. when scope was wide). This scope effect was smaller for postonomials than prenominals $(\mathrm{BF}=16$ for the Scope by Modifier-Position interaction. Subsequent pairwise comparisons confirmed that there was strong evidence for an effect of Scope for prenominals $(\mathrm{BF}>100)$ but not for postnominals $(\mathrm{BF}<0.2)$. 


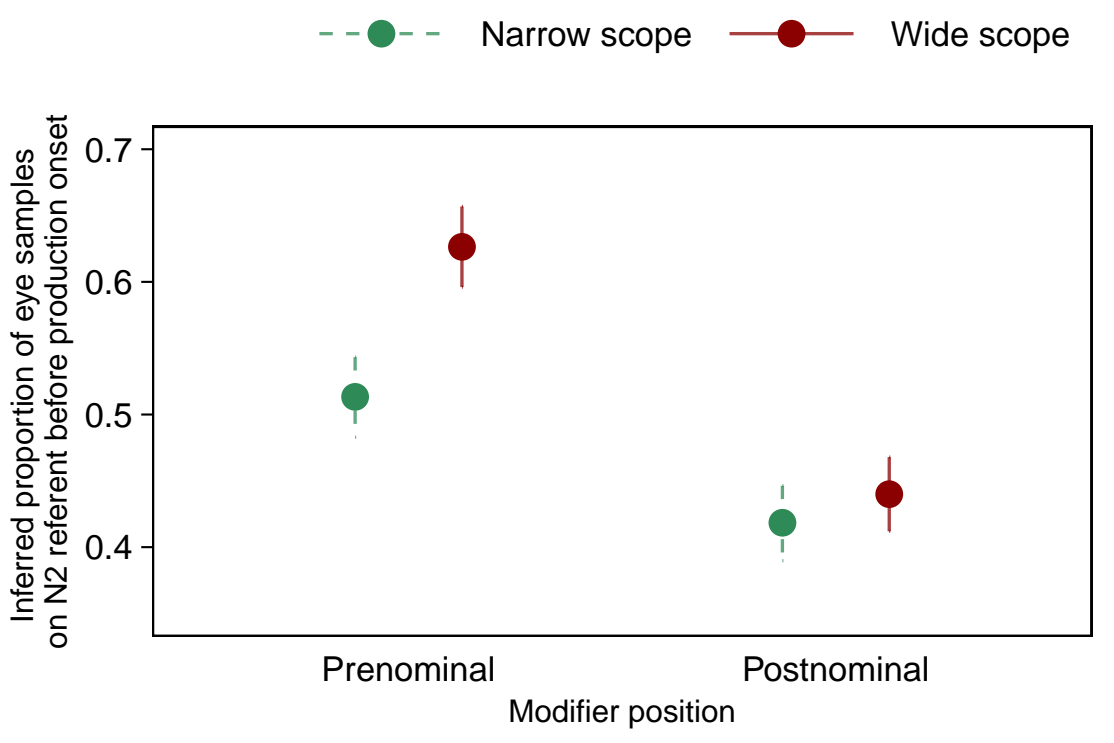

Figure 2

Summary of the parameter values for the proportion of eye samples on N2 before production onset inferred from the Bayesian linear mixed effects model. The figure shows the posterior distributions of the parameter values with the maximum a posteriori parameter value $\hat{\mu}$ and 95\% PIs (Experiment 1).

\section{Onset latency}

The onset latency was transformed to the reciprocal of its square root as suggested by a Box-Cox test (Box \& Cox, 1964). The transformed onset latency was fitted with main effects of Modifier Position, Scope and their interaction.

Figure 3 gives estimated cell means from this model. There was weak evidence $(\mathrm{BF}=2.4)$ for a main effect of Scope with longer latencies for wide scope. Unlike eye data effects there was negligible evidence for a Scope by Modifier Position interaction $(\mathrm{BF}=1.1)$. Pairwise comparisons gave weak evidence for longer onset latencies for wide scope in prenominals $(\mathrm{BF}=2)$ but no evidence for an effect in postnominals $(\mathrm{BF}=0.5)$. 

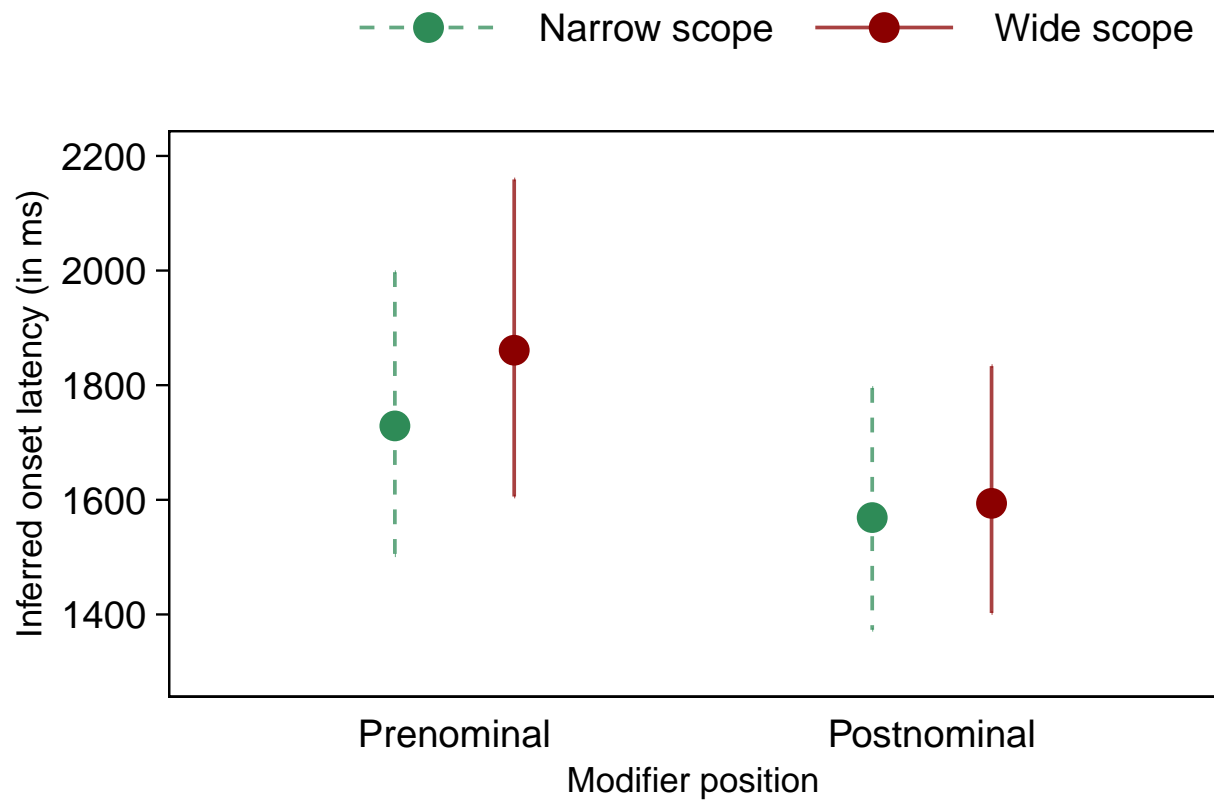

Figure 3

Summary of inferred onset latency for Scope by Modifier position inferred from the Bayesian linear mixed effects model. Shown are the maximum a posteriori parameter value $\hat{\mu}$ and $95 \%$ PIs (Experiment 1).

\section{Discussion}

Experiment 1 suggests that semantic relation can determine scope of advance planning in subordinated noun phrases when surface - and therefore syntactic - form is held constant. Specifically semantic contrast led to increased planning of non phrase-initial nouns, in phrases with a prenominal modifier expressing possession. In these phrases the proportion of eye samples on the N2 referent increased if $\mathrm{N} 2$ had a contrastive function. There was also weak evidence that this effect was associated with longer production onset latencies.

The fact that the scope effect was present for prenominal but not for postnominal modifier phrases indicates that, in the context of this experiment, scope was moderated by one or more other factors. It is possible that, as we hypothesized, decision about 
pre-planning scope is dependent on the structural function of the phrase-initial noun (Brown-Schmidt \& Konopka, 2015) in both the prenominal and postnominal conditions, but only if the participant has identified the presence of two images: the semantic structure (i.e. determiner scope) can only be determined if the participant apprehends both referents in the first place. In Experiment 1 the colour of the target attracted early fixations during screen apprehension. In the prenominal modifier condition participants would then need to fixate the N1 referent in order to start output, thus forcing apprehension of both images. In the postnominal modifier condition the coloured image represented N1 and therefore N1 and its determiner could be output without any requirement to apprehend the N2 referent.

Arguably one limitation of Experiment 1, and a possible contributing factor in our failure to find scope effects in the postnominal modifier condition, is that the absence of an explicit addressee meant that participants lacked explicit communicative purpose. There was no obvious communicative motivation for participants to process the semantic scope of the modifier. Findings from spoken discourse show that people tend to produce less precise referential phrases in the absence of an interlocutor (Van Der Wege, 2009). Experiment 2 therefore introduced an addressee.

\section{Experiment 2}

Experiment 1 showed support that semantic scope affects pre-planning. The scope effect was found in prenominal noun phrases but not in postnominals.

To increase salience of semantic scope Experiment 2 used an interactive image-description task (see e.g. Brown-Schmidt \& Konopka, 2008, 2015; Brown-Schmidt \& Tanenhaus, 2006; E.-K. Lee et al., 2013; Swets, Jacovina \& Gerrig, 2013, 2014). Participants were required to unambiguously identify one of several images to the experimenter, who then responded by pointing to the image that they communicated. As in Experiment 1, uniquely identifying images required production of noun phrases in which either the first or second noun served a contrastive function. 
In contrast to Experiment 1 participants were free to choose whether to use a postnominal phrase (The painting with the man) or prenominal phrase (The man's painting) to describe the target image. In Experiment 1 participants had to remember that a vertical line indicates that they need to produce prenominal structures. This potentially induced additional difficult in the naming task. Allowing free selection of which phrase participants want to use makes the task easier and more natural. To ensure that participants were aware of the semantic scope required for the target phrase, every target trail was preceded by a priming trial that served to make salient the particular contrast represented by the target.

As in Experiment 1, participants provided written responses, for the reasons discussed in the Introduction: there is no pressure for fluent utterances in writing which might confound results reported for spoken production experiments; and writing allowed us to keep the (prosodic) surface form in the produced utterances constant across scope condition. In Experiment 2 we included a spoken condition. Although our hypotheses are modality-independent and, as we have argued, writing provides a robust context in which to test them, previous research has focused more or less exclusively on spoken production. Replication in speech would, therefore, allow a more direct comparison with previous findings.

In line with Experiment 1, we predicted that participants are more inclined to pre-plan the second noun (N2) in the target phrase. Increased gaze on the N2 referent image and longer onset latencies would both evidence an increased tendency to pre-plan N2. As the manipulated scope contrast is prelinguistic, we predicted this effect in both prenominal and postnominal phrases, thus regardless of the syntactic configuration of the target phrase and the arrangement of the target array. We predicted similar effects in writing and in speech. Again, the surface form - the syntactic structure and the lexical content - was held constant across scope conditions. 


\section{Method}

\section{Participants}

Sixty-four psychology students (57 female, 7 male, mean age $=20.4$ years, $S D=5.4$, range: $18-50)$ participated as part of a research-reward scheme. All participants were self-reported native speakers of British English, free of linguistic impairments, and had normal or corrected-to-normal vision. Five participants were replaced because of a combination of failure to follow task instructions or failure to obtain well calibrated eye data.

\section{Design EG Materials}

Participants were presented with arrays containing two image pairs and four other images (Figure 4). Image pairs were linked by a vertical line with the target image always appearing below its modifier.

Participants had to instruct the experimenter to click on the highlighted image using prenominals or postnominal structures. Participants were allowed to choose noun modification freely, guided by examples provided in instructions at the start of the experiment. As can be seen from Figure 4 determiner scope was dependent on choice of phrase structure.

To make scope salient we used a prime-target design. Across consecutive trials, using that same array of images, participants first produced a noun phrase based upon one image pair (the prime; indicated by the dotted circle), and then on another image pair (the target trial, for which we report data; indicated by the solid circle). This is illustrated in Figure 4. The prime pairs, in these examples, are shown in the bottom right corner. N1 and N2 served the same contrastive functions in both prime and target. So noun phrases required to express prime and target were semantically isomorphic (and syntactically identical). Each participant completed a written and a spoken session. These manipulations rendered a narrow scope/wide scope $\times$ prenominal/postnominal $\times$ written/spoken design. 

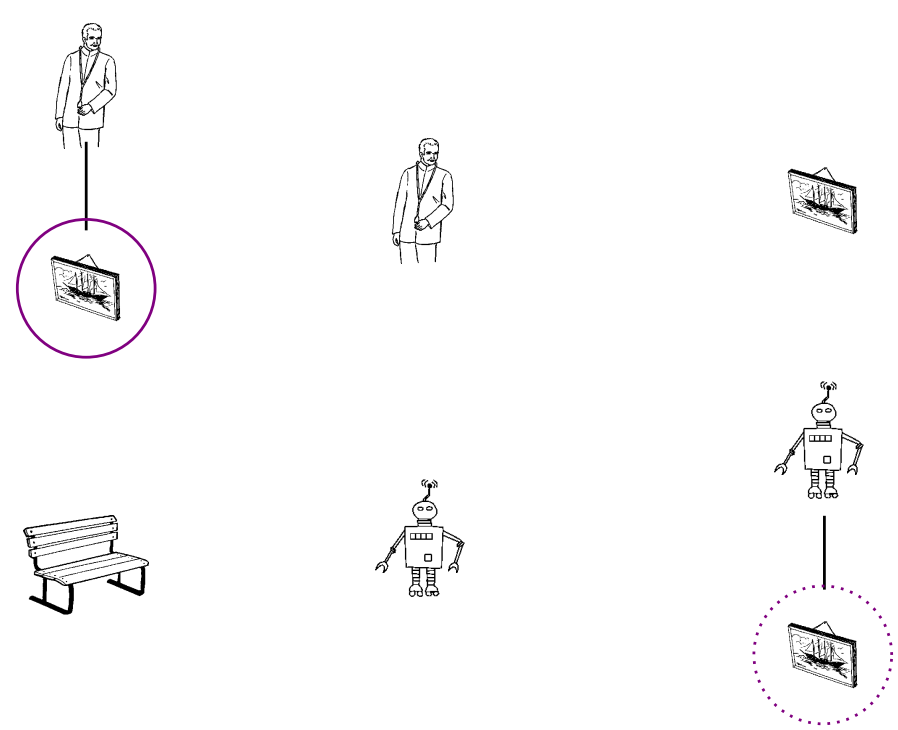

(a) The man's painting (narrow scope) / The painting with the man (wide scope) land not the painting with the robot]
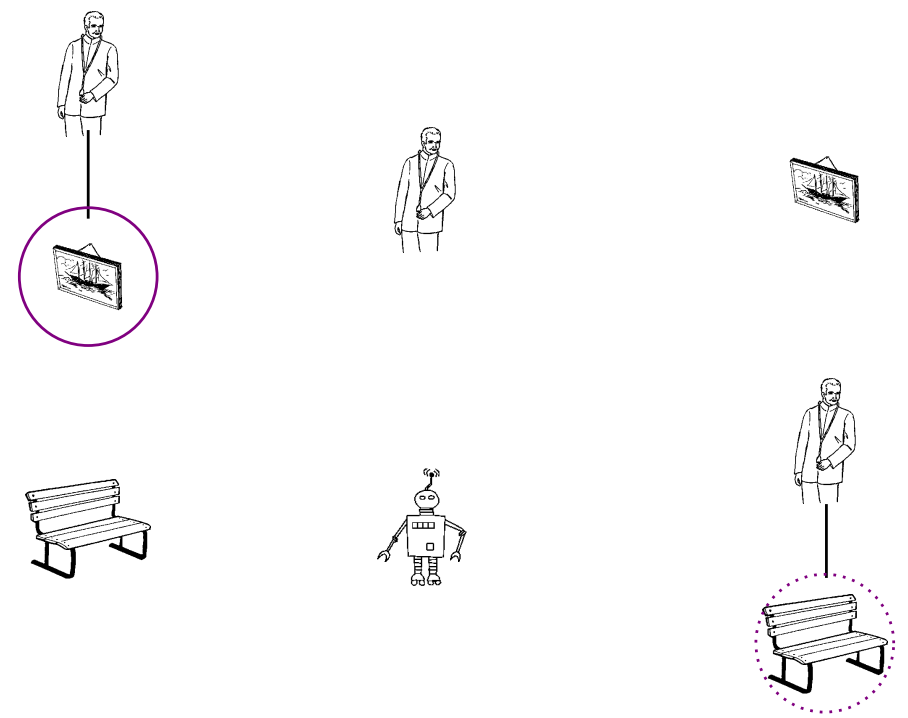

(b) The man's painting (wide scope) / The painting with the man (narrow scope) land not the bench with the man]

\section{Figure 4}

Example stimulus arrays (Experiment 2). Participants produced phrases as indicated, uniquely identifying the circled image, having first produced a similar phrase (the prime) based on the same array and uniquely identifying the image here shown with a dotted circle. 
Thirty-two item screens were created including the two conditions shown in Figure 4. In each trial participants were presented with an array with six cells. One cell included the target image pair and one cell the prime image pair (see Appendix B for a full list of the stimulus material). The remaining four cells were filled with the four images used for the target and the prime pair. Thus modification of the target phrase was always required. This array was first used to present the prime and then to present the target. Items were counterbalanced over four Latin Square lists (Scope, Modality Type [speech, writing]). Order of modality session was counterbalanced between participants; half of the participants started with a written session and the other half with a spoken session. The location of prime and target pairs was randomised within each Latin square list. Two filler lists each of 16 trials were created. One filler list was used in the first session and the other the second session. Fillers elicited target structures different form the stimuli to avoid strategic use of descriptions and anticipation of the target pair. For example, the top image of image pairs were prompted, and colour (in combination with modifiers) was used for disambiguation (e.g. the cat's green ball). Filler lists and stimuli were presented in randomised order within session. In total, each participant saw 64 arrays, 32 per modality (50\% fillers).

We calculated two variables from the eye data: (1) the time - relative to production onset - for when gaze shifted from the image of N1 to N2; (2) the proportion of trials for which this gaze shift happened before production onset. Gaze shift from the N1 referent to $\mathrm{N} 2$ was defined as the first fixation on the image of N2 after the gaze left N1 (fixation required a dwell time of at least $100 \mathrm{~ms}$ ). These measures estimate the attention shift from the referent of the first noun to the preparation of the second noun (see e.g. Griffin, 2004; Griffin \& Bock, 2000; Konopka \& Meyer, 2014; Meyer \& Lethaus, 2004). 


\section{Apparatus \& Procedure}

The apparatus was the same as in Experiment 1, with the addition of a Logitec headset on ASIO audio driver supported by the Creative SB X-Fi sound card for recording spoken output, and an additional display and a mouse to allow experimenter response.

Every experiment started with camera set-up, and 9-point calibration and validation. Participant and experimenter were seated at different screens and were unable to see each other's screen. Each prime / target trial pair was initiated by the participant fixating an ellipsis at the centre of the screen (as in Experiment 1). Recalibration was performed if this fixation trigger failed. The stimulus array then appeared, with a violet circle highlighting the image that was the target of the prime trial. The participants spoke or wrote a noun phrase intended to uniquely identify the image, and then pressed enter. This activated mouse input for the experimenter who then clicked on the image identified by the participant. For ambiguous utterances, the experimenter clicked an image that was a plausible referent. Mouse clicks on the correct image prompted a green circle as feedback. Clicks on an incorrect image prompted a red circle. Feedback circles were shown for $250 \mathrm{~ms}$. The second image (the target) was highlighted immediately after the feedback circle disappeared. The target trial then proceeded in the same way as the prime trial.

Participants were familiarised with the experimental task in 10 practice trials. The images, size $150 \times 150$ pixels, appeared around the centre of the screen. In the written condition participants gave their response in a text box $(896 \times 50$ pixels $)$ that appeared in the centre of the screen. The array remained on the screen while they typed. Participants could pause, if they wished, between trial pairs. The duration of the experiment was approximately 45 minutes.

\section{Results}

Prior to analysis we removed trials with extremely long pauses ( $>2.5$ secs), ambiguous descriptions and self-corrections (9.9\%). Trials in which either or both the 
prime and target response are ambiguous were excluded (e.g., trials where the participants gave different modifier positions in response to the prime and to the target; $5.6 \%)$. Onset latencies $>10$ secs were removed $(0.3 \%)$. For the analysis of eye data, we removed a further $2.4 \%$ of the trials which had more than $75 \%$ of the eye fixations away from any of the images in the array, before production onset. The critical AOIs for analysis were the images corresponding to the first and second noun in the produced utterance. As in Experiment 1, the size of the AOI was equal to the size of the images. Statistical analysis followed the same methods as for Experiment 1.

Participants were free to choose prenominal or postnominal noun modification. However, as can be seen from Table 1 modifier-position choice was independent of scope. A full-factorial Bayesian logistic linear mixed effects model found negligible evidence for a Scope effect on modifier type (prenominal or postnominal) or any by-Scope interactions $(\mathrm{BF}<0.5)$. Participants were roughly equally likely to choose prenominal and postnominal constructions in the written modality, but showed a greater tendency towards postnominals when speaking ( $\mathrm{BF}>100$ for the main effect of modality).

\section{Table 1}

Proportion of trials on participants' produced a postnominal modifier (The man with the picture) in preference to a prenominal modifier (The man's picture). Estimates $\hat{\mu}$ from a full-factorial linear mixed effects model with maximal random effects structure. 95\% PIs in parenthesis (Experiment 2).

\begin{tabular}{llcc}
\hline Prime/Target & Scope & Writing & Speech \\
\hline Prime & Narrow & $.47[.34, .59]$ & $.68[.55, .81]$ \\
& Wide & $.49[.36, .61]$ & $.70[.57, .83]$ \\
Target & Narrow & $.47[.34, .58]$ & $.69[.55, .81]$ \\
& Wide & $.48[.35, .60]$ & $.70[.56, .83]$ \\
\hline
\end{tabular}

We established the effect of determiner scope on each of the three dependent 
variables. From the eye data we calculated the time relative to production onset when gaze shifted from the image representing the first noun to the image representing the second noun in the phrase that was produced. We took this as indication of attention shift from the first to the second referent. Second, we evaluated the probability that this shift occurred prior to the participant starting to output (speak or write) the phrase. Finally, we calculated onset latency - time from appearance of the circle prompt to output onset.

Analysis was by Bayesian linear mixed effects models for continuous variables (gaze shift and onset latency) and by generalised logistic mixed effects models for the probability of gaze shift before output onset. All models were fitted with main effects and all interaction effects of Modality (written, spoken), prime/target, Modifier position (prenominal, postnominal), and Scope (wide, narrow). As before, all models were fitted with the maximal random effects structure; random intercepts for participants and items and by-participants and by-items slope adjustments for all fixed effects. We then conducted a series of eight planned comparisons, based on posteriors from these models, to test for evidence of advance planning in the wide scope condition, relative to narrow scope within each cell of the Modality by Modifier position by prime/target interaction.

We present findings from the overall model first. Then, for clarity, we report effects in just target trials, first in the written modality, then in the spoken modality. For completeness, effects in the prime trails are summarised in Appendix C.

Findings from the main model were as follows. In $97 \%$ of trials gaze shift from the first to second noun referent was detected either before or after production onset. For statistical analysis the data were shifted above zero to allow log transformation to correct for positive skew (Box \& Cox, 1964). We found strong evidence for a main effect of Scope $(\mathrm{BF}>100)$ showing earlier gaze divergence for wide scope. The evidence for all by-Scope interactions was weak $(\mathrm{BF}<2)$. The analysis of the probability that gaze shift to the N2 referent occurred before production onset provided strong evidence of a Scope by Modifier-position by Modality interaction $(\mathrm{BF}=8.5)$ and evidence of a Scope by 
prime/target interaction $(\mathrm{BF}>100)$. These effects are unpacked in the comparisons described below. Response onset latency was log transformed prior to modelling to correct for positive skew. We found strong evidence for a main effect of Scope $(\mathrm{BF}=68)$, and moderate support for a Modifier position by Scope interaction $(\mathrm{BF}=3.5)$. There was no evidence for other effects $(\mathrm{BF}<2)$ across any of the three dependant variables.

\section{Written output modality}

Estimated mean time of gaze shift from the N1-referent image to the N2-referent image is shown in Figure 5a. This shows earlier gaze on N2 under wide scope for both prenominal and postnominal modifiers (evidence for effect of scope: prenominal $\mathrm{BF}=15$; postnominal $\mathrm{BF}=23$ ). In fact for postnominal modifiers gaze shift in the wide scope condition tended to occur before production onset but not in the narrow scope condition. For prenominal modifiers, gaze shift was observed after production onset but earlier for the wide scope condition.

As might be expected, the probability of gaze shift to occur before production onset followed the same pattern. Estimated probability was greater in the wide scope condition both for phrases with prenominal modifiers and with postnominal modifiers (prenominal: narrow-scope $\hat{\mu}=.19,95 \%$ PI $[.13, .26]$, wide-scope $\hat{\mu}=.39,95 \%$ PI $[.31, .5], \mathrm{BF}=95$; postnominal: narrow-scope $\hat{\mu}=.27,95 \%$ PI $[.19, .35]$, wide-scope $\hat{\mu}=.55,95 \%$ PI $[.45, .65], \mathrm{BF}>100)$.

The inferred posterior parameter values for the onset latency are visualised in Figure 5b. The results show that production onset latency was shorter under wide scope for postnominal modifiers $(\mathrm{BF}=12)$ but not for prenominal modifiers $(\mathrm{BF}<0.05)$. 


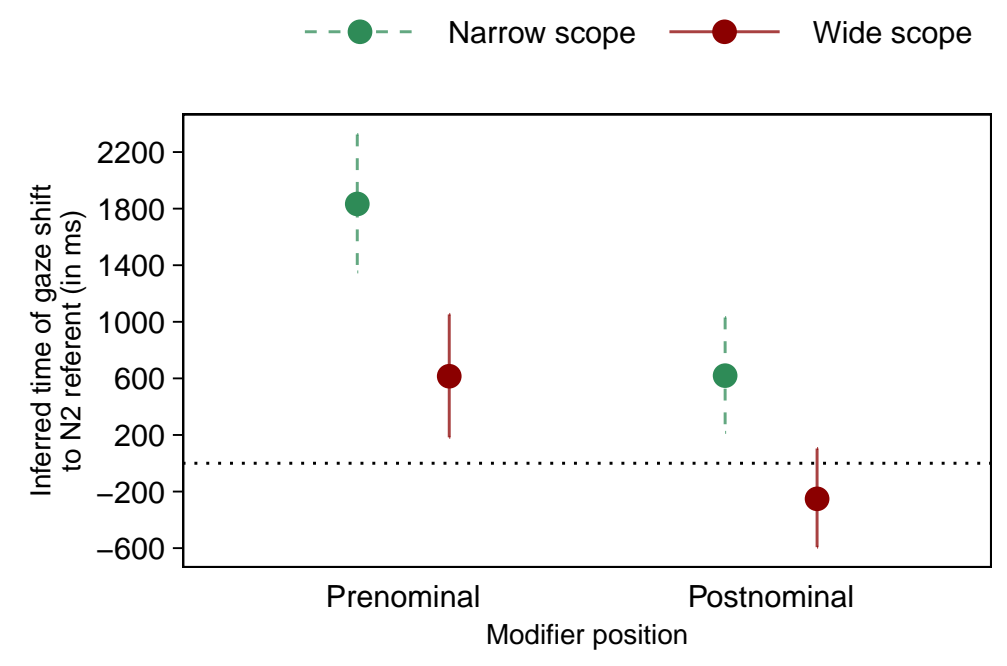

(a) Time of gaze shift from N1 to N2 referent relative to

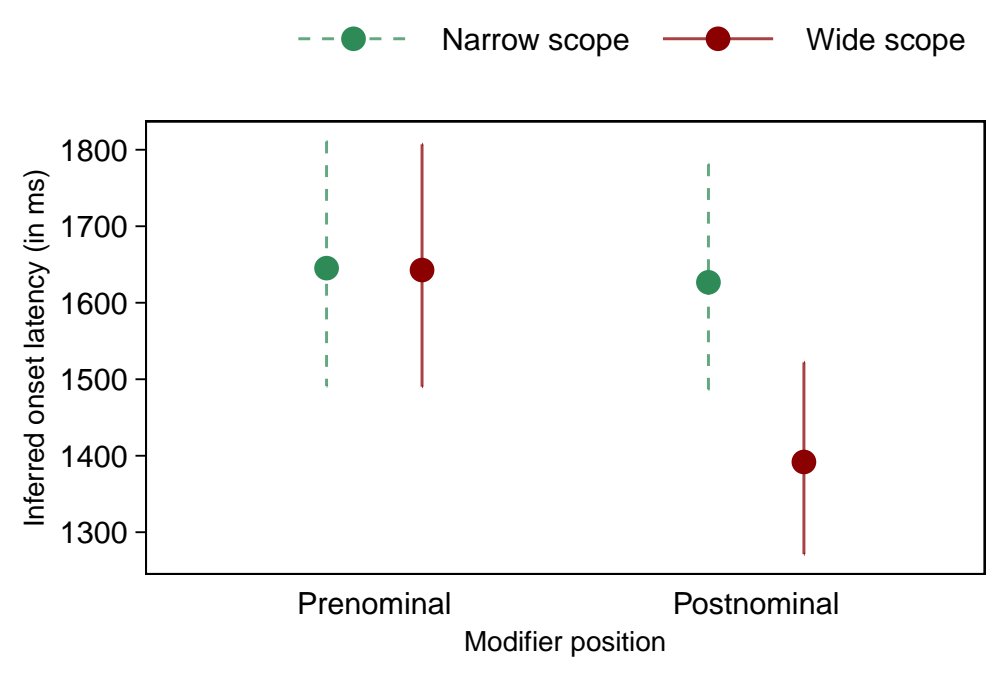

(b) Circle prompt to production-onset latency.

production onset. The dotted line indicates the production onset.

Negative values indicate time before production onset.

\section{Figure 5}

Experiment 2, Written production. Mean gaze shift and production onset latencies. Error bars represent $95 \%$ PIs. Parameter estimates from full-factorial Modality $\times$ prime/target $\times$ Modifier-position $\times$ Scope Bayesian linear mixed effects models. 


\section{Spoken output modality}

Estimated mean time of gaze shift from the N1-referent image to the N2-referent image is shown in Figure 6a. Although parameter estimates suggest earlier gaze-shift under wider scope for postnominal modifiers we found negligible evidence for this effect in either modifier position $(\mathrm{BF}<0.08)$. We found strong evidence indicating that the probability that gaze shifted from the N1 referent to the N2 referent before production onset was greater in the wide-scope condition in postnominals (narrow-scope $\hat{\mu}=.51,95 \% \mathrm{PI}$ $[.42, .6]$, wide-scope $\hat{\mu}=.81,95 \%$ PI $[.74, .87], \mathrm{BF}>100)$. However, this effect was absent in prenominals (narrow-scope $\hat{\mu}=.4,95 \% \mathrm{PI}[.29, .52]$, wide-scope $\hat{\mu}=.5,95 \% \mathrm{PI}$ $[.38, .63], \mathrm{BF}<0.8)$. These findings therefore reproduce Scope effects found in written production for phrases with postnominal modifiers but not for prenominals.

As can be seen from Figure 6b, we found strong evident that production onset occurred sooner under under wide scope when participants produced phrases with postnominal modifiers $(\mathrm{BF}=26)$ but not when modifiers were prenominal $(\mathrm{BF}=0.11)$. This is the same pattern as was found for written production. 


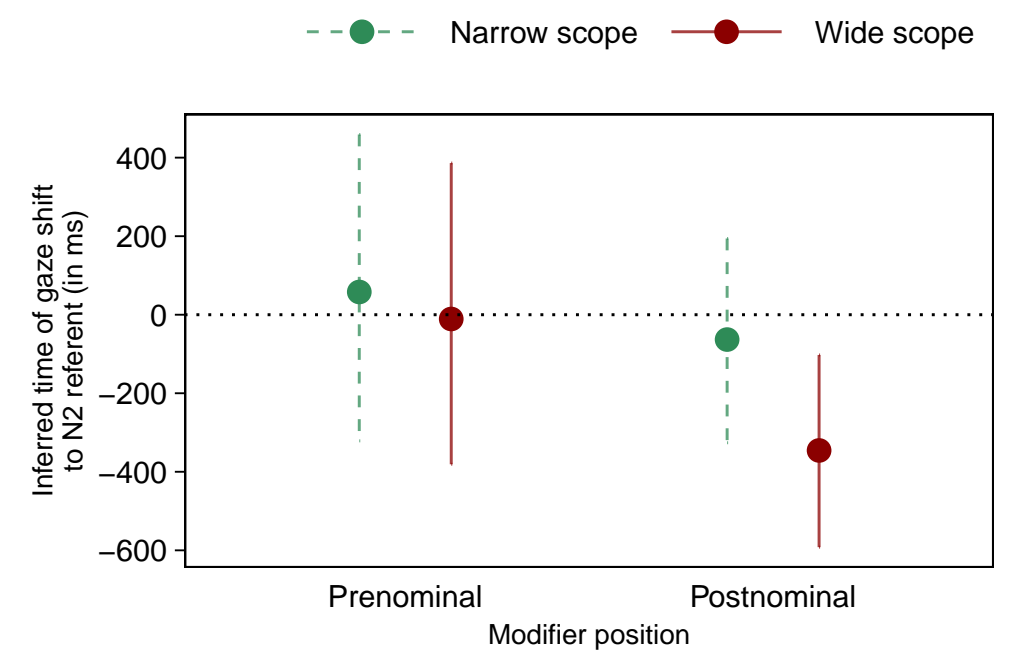

(a) Time of gaze shift from N1 to N2 referent relative to

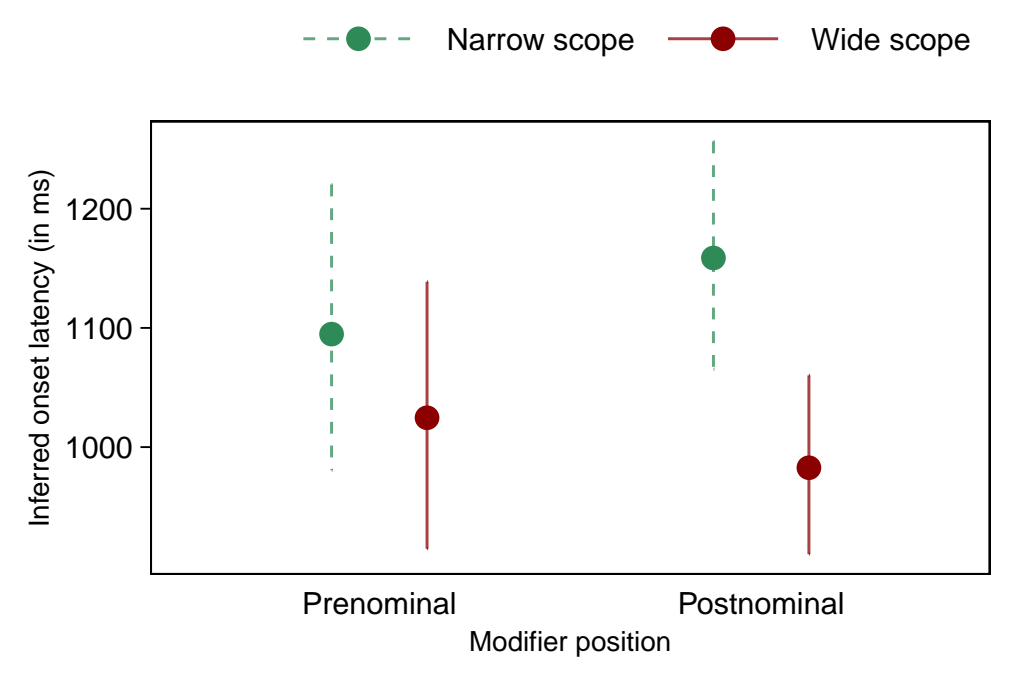

(b) Circle prompt to production-onset latency.

production onset. The dotted line indicates the production onset.

Negative values indicate time before production onset.

\section{Figure 6}

Experiment 2, Spoken production. Mean gaze shift and production onset latencies. Error bars represent 95\% PIs. Parameter estimates from full-factorial Modality $\times$ prime/target $\times$ Modifier-position $\times$ Scope Bayesian linear mixed effects models. 


\section{Discussion}

As in Experiment 1, findings from Experiment 2 suggested that the (semantic) contrastive relationship between the phrase elements influenced processing of the N2 referent (i.e. the referent of the second noun in the output phrase). This occurred despite the fact that surface form output was held constant for wide and narrow scope contrasts. Gaze shift to N2 was earlier and more likely to occur before production onset if N2 had a contrastive function for the phrase's reference. This effect was found across modality for phrases with postnominal modifiers and just in writing for prenominal phrases. This indicates, we believe, that the semantic structure of non phrase-initial nouns influences advance planning even when holding the syntactic form and the lexical content of the phrase constant. Evidence for this effect was found in both writing and in speech data. This suggests that the scope effect cannot be explained in terms of modality-specific processing demands alone. Instead, the Scope effect is consistent with a fundamental modality-independent process underlying language planning.

However, there were some differences in effects across modality and, particularly, direction of the scope effect in response latency in postnominal phrases was the reverse of our prediction. We address these issues in the remainder of this discussion.

Evidence for earlier planning of non phrase-initial nouns comes from the eye movement data. We found that in writing gaze shift to the referent of N2 was earlier under wide scope and more likely to occur before production onset, regardless of modifier position. For speech there was a higher probability to shift gaze to the second noun before production onset under wide scope when planning postnominal phrase but not when planning prenominal phrases (although, as we discuss below, these were only chosen by participants in $30 \%$ of cases; see Table 1 ).

This difference between writing and speech was not anticipated but might be explained as follows: As we discussed in the Introduce there is, in principle, no pressure to maintain output fluency in writing. Pre-planning is a compromise between mental 
buffering of pre-planned items and intra-utterance pausing once speaking commences (Levelt \& Meyer, 2000; Meyer, 1997). This pressure exists in speech, particular when, as was the case in Experiment 2, the utterance must communicate the speaker's message rapidly and accurately to a real and present listener. Maintaining this fluency requires some anticipation of environmental demands on the production process (Griffin, 2003; Griffin \& Bock, 2000). To reduce pre-planning demands, speakers may assess the possibility of lexical retrieval in parallel to articulation (Allum \& Wheeldon, 2007; Griffin, 2003). If there is insufficient time between the onset of the first noun $\mathrm{N} 1$ and the onset of the second noun N2 to retrieve the lexical item N2, speakers may pre-plan N2 before the onset of N1. Failure to do this would mean that speakers would have to pause prior to N2 onset, which has, at least in some contexts, potential effects on what the utterance communicates (e.g. Clark \& Fox Tree, 2002). There is more time between N1 output and $\mathrm{N} 2$ output in postnominal phrases - N1 with the - than in prenominals - N1's. It may be, therefore, that fluency requirements encourage pre-planning in prenominal modification, thus masking effects of semantic scope, but that this does not occur in the case of the less time-pressured speaking of phrases with postnominal modifiers.

This account is consistent with participants' preference, when speaking but not when writing, for postnominal rather than prenominal modifiers. Further analysis suggested that, for speech, modifier choice (pre- vs. postnominal) was indeed reliably different from what we would expect if a priori, at $50 \%$ chance-level, either structure was equally likely $(\hat{\mu}=.7,95 \% \mathrm{PI}[.56, .82], \mathrm{BF}=55)$. This was not the case for writing $(\hat{\mu}=$ $.48,95 \% \mathrm{PI}[.35, .6], \mathrm{BF}=0.9)$. This preference for postnominal modification in speech might be explained in terms of planning efficiency. To reduce pre-planning demands our participants may have preferred postnominal modification in speech over prenominal modification. Such a preference did not emerge in writing.

Eye data provide evidence for non-linear planning in wide scope conditions. In line with the sentence planning literature (e.g. Martin et al., 2010; Wheeldon et al., 2013) it 
might be expected that increased planning scope would lead to longer response-onset latencies because language planning is process-demanding. However, we did not find longer latencies in wide-scope conditions and, in fact, found that they were reliably shorter for wide-scope postnominals.

We return in our general discussion to broader issues around scope and onset latency. The present finding of shorter onset latencies for wide scope when the output phrase involved a postnominal modifier is explained, we believe, by factors that were a necessary feature of the stimulus arrays. To make salient the contrastive reference in the target noun phrase, participants first produced a prime phrase. In the wide scope condition the first noun in the phrase was the same in the prime and target utterance (prime: the painting with the robot; target: the painting with the man). In the narrow scope condition, the second noun was the same (prime: the bench with the man; target: the painting with the man). This meant that when participants came to plan their target phrase in just the wide-scope condition the initial noun was strongly primed. However, shorter onset latencies for wide-scope conditions was only found in the longer postnominal phrases and not in the (shorter) phrases with prenominal modification. This finding is analogous to that found by Griffin (2003). She observed shorter onset latencies for noun pairs if the first noun was long compared to noun pairs in which the first noun was short. She explained this in terms of

parallel planning after production onset; the second noun could only be planned in parallel to production if uttering the first noun provided enough time.

\section{General Discussion}

Previous studies exploring pre-planning of phrases or short sentences have concluded that the minimum size of the linguistic units in the surface structure of the text or utterance that must necessarily be prepared prior to output-onset is determined by the syntactic dependencies between phrase elements (E.-K. Lee et al., 2013; Martin et al., 2010; Nottbusch et al., 2007; Roeser et al., 2019; Smith \& Wheeldon, 1999; Swets et al., 
2014; Wagner et al., 2010; Wheeldon et al., 2013). The aim of this investigation was to test the hypothesis that the extent to which phrases must be pre-planned is in fact driven by semantic dependencies in the message to be communicated acting prior to and independently of any syntactic or lexical processing.

Our findings, with some caveats that we discuss below, suggest that for the production of noun phrases, this is in fact the case. We found that non-phrase-initial nouns that had a contrasting function required earlier processing during the planning process. Eye movement in Experiment 1 indicated that participants gave more attention to the referent of the second noun when it served a contrastive function (i.e. in the wide-scope condition). Experiment 2 confirmed that if the second noun had a contrastive function gaze-shift to the referent of the second noun was initiated earlier and was more likely to happen prior to production onset. This suggests that pre-planning contrastivity requires early attention to what lies beyond the first determiner-noun pair.

This conclusion implies that pre-planning for noun phrases might generally be determined at the semantic level. As we have noted, this is in contrast to the conclusions of previous researchers who have argued that the syntactic structure of the noun phrase determines the extent of obligatory advance planning (E.-K. Lee et al., 2013; Martin et al., 2010; Roeser et al., 2019; Smith \& Wheeldon, 1999). A difficulty for this account, however, is that it is not clear how the production system "knows" the size of the to-be-encoded syntactic unit prior to grammatical encoding. If the full syntax tree must be planned before the processor can establish that scope could be narrow, there is clearly then no timing advantage to be gained from the knowledge. When preparing complex noun phrases that are larger than a determiner-noun pair the production system must decide planning extent prior to syntax-structure. This is essential if advance planning is to be less extensive, and therefore less time-consuming, for simple - as opposed to co-or subordinated - noun phrases (e.g. Allum \& Wheeldon, 2007, Nottbusch et al., 2007, Smith \& Wheeldon, 1999, Wheeldon et al., 2013 and similarly for noun phrases with structurally non-adjacent 
dependencies E.-K. Lee et al., 2013).

We found that the semantic relation between phrase elements affects pre-planning of non-initial noun referents. Establishing this relation is fundamental for grammatical encoding of the phrase and thus, semantic planning - and not grammatical encoding determines the scope of pre-planning noun phrases. The scope of grammatical encoding, therefore, might be determined on a semantic level of representation rather than by syntactic relations between phrase elements. In other words, semantic relations between phrase elements are fundamental for planning beyond the phrase-initial determiner-noun pair. In the remainder of this section we address two caveats and suggest possible implications of our findings that are potential foci for future research.

The first is that the scope effect in Experiment 1 (writing) was present for phrases where the modifier occurred before the modified noun but not where the modifier was postnominal. In Experiment 2 we observed that under wide scope participants shifted their gaze from N1 to N2 before production onset in postnominal phrases (in both modalities) but after production onset in prenominal phrases in writing and not at all in speech. These differences suggest that both the interactivity of the task (i.e. the presence of an interlocutor) and output modality affect planning on a semantic level. We elaborate on these factors in turn.

Regarding the interactivity of the task, if we assume that the generation of semantic relations is required by the need to uniquely identify a target referent (Brown-Schmidt \& Konopka, 2008; Brown-Schmidt \& Tanenhaus, 2006; E.-K. Lee et al., 2013; Swets et al., 2014) then our failure to observe this effect for postnominal phrases in Experiment 1 can, perhaps, be explained by the absence of an interlocutor. As there was no interlocutor in Experiment 1, there is no communicational need to allocate planning resources to the identification of the correct target. As for the scope effect observed for prenominal phrases, it is worth noting that, in the present experiments, stimulus apprehension is initially guided by a visual cue (colour in Experiment 1; a circle in Experiment 2). The cued image 
is the second noun in a prenominal phrase but the first noun in a postnominal phrase. This is important as language planning is known to build on an interaction of both perceptual features and linguistic preferences (Gleitman et al., 2007; Kuchinsky, 2009; Kuchinsky et al., 2011; Myachykov, Garrod \& Scheepers, 2012; Tomlin, 1995). While both phrase referents must be fixated before production onset in prenominals, this is not the case in postnominal phrases; looking at the modifier referent was not cued and is not necessarily obligated by the production system if encoding the first noun suffices to release production onset. Yet, we found that under wide scope, gaze was shifted to N2 before production onset in postnominal phrases which we observed in the interactive set-up (Experiment 2).

This does not, however, explain why we observed scope effects for prenominal modifiers in writing (Experiment 1 and 2) but not in speech (Experiment 2). One possible explanation is that modality-specific factors masked the scope effect. Pre-planning in speech must involve some anticipation beyond the first noun to ensure output fluency (Griffin, 2003). For example, speakers need to anticipate whether or not the second noun can be planned in parallel to articulation as pausing during the production process may change the meaning of spoken utterances (e.g. Clark \& Fox Tree, 2002). There is not the same fluency requirement in writing. In the present study, there was less time to plan the second noun in parallel with articulation of the first noun in prenominal phrases (The N1's ...) relative to postnominal phrases (The $N 1$ with the ...). So scope effects for prenominal phrases might have been eliminated in speech for modality-specific reasons: planning of the second noun was required to ensure output fluency after production onset.

A second potential caveat is the following: previous studies that sought to determine the minimal unit of advance grammatical encoding in noun phrases drew on evidence from stimulus-to-onset latency (E.-K. Lee et al., 2013; Martin et al., 2010; Smith \& Wheeldon, 1999; Wheeldon et al., 2013) with effects mirrored in participants' eye-movements (Roeser et al., 2019). In the present study onset latency data gave only weak (Experiment 1) or no evidence (Experiment 2) for increased planning demands under 
wide scope. ${ }^{2}$ As with studies that have focused on conceptual planning (Brown-Schmidt \& Konopka, 2008; Do \& Kaiser, 2019; Konopka \& Meyer, 2014; Swets et al., 2014), our conclusions are supported by eye-movement data. It is worth noting that research that has tapped into conceptual processing has tended to find effects on eye-movement but not on onset latency. These studies characterised pre-planning of conceptual representations as the product of a very rapid apprehension of the stimulus screen (Griffin, 2004; Griffin \& Bock, 2000; Konopka \& Brown-Schmidt, 2014; Meyer \& Lethaus, 2004) involving an efficient allocation of processing resources (Swets et al., 2014). In other words, pre-planning on the conceptual level may affect how gaze is used to encode information but does not add to the pre-planning demands associated with grammatical encoding (Gleitman et al., 2007; Griffin \& Bock, 2000; Konopka \& Meyer, 2014). This is in contrast to the idea that pre-planning demands originate on a conceptual level (F. Ferreira \& Engelhardt, 2006) and activation cascades automatically into grammatical encoding (Bock \& Ferreira, 2014; Fodor, 1983; Kempen \& Hoenkamp, 1987; Levelt, 1989). Instead our data, in conjunction with other studies cited above, suggest that semantic planning of non phrase-initial elements may not immediately activate lexical or syntactic representations. This is because we would expect that onset latency increases only when the semantic plan cascades into grammatical encoding. If this is true, then it would suggest that onset-latency effects found by E.-K. Lee et al. (2013) and other investigations concerned with the minimal unit of advance planning (e.g. Martin et al., 2010; Smith \& Wheeldon, 1999; Wheeldon et al., 2013) are syntactic / lexical by nature rather than semantic.

We now turn to consider implications of our findings. Our findings suggest that relational dependencies are fundamental for planning beyond the first determiner-noun pair. We distinguish between conceptual and semantic representations (which, are often used interchangeably in psycholinguistic production models; Momma et al., 2015, p. 8). In

\footnotetext{
${ }^{2}$ In fact, we observed a speed-up effect for wide scope in postnominal noun phrases that we addressed in the Discussion of Experiment 2.
} 
our experiments determining the unique identifiability of an image was the central requirement for pre-planning contrastivity. For the production system to decide whether or not a referent is uniquely identifiable requires (a) the recognition of the target referent and (b) determining whether or not a referent with conceptually similar properties is present and, eventually, (c) deciding the properties that can be used to distinguish the target referent from a contextually available competitor. While the first two steps are conceptual, only the last step is related to the semantic structure of the target utterance. It remains unclear, however, exactly how conceptual and semantic representations interact during language planning.

There is compelling evidence that encoding structural relations relies on semantic representations (Bunger et al., 2013; Do \& Kaiser, 2019; Konopka \& Kuchinsky, 2015; Konopka \& Meyer, 2014; Momma \& Ferreira, 2019; Momma et al., 2015). A question that recently received attention in the literature is to what extent conceptual encoding is non-relational - identifying concepts and then building structure - or relational processing relationships between entities before the entities themselves (Konopka \& Bock, 2009; Konopka \& Brown-Schmidt, 2014; Konopka \& Meyer, 2014; Kuchinsky, 2009). These studies attribute non-linear planning to the activation of a conceptual-thematic representation of the verb and its argument(s) (Bock \& Ferreira, 2014; Bock \& Levelt, 1994; Chang et al., 2006; F. Ferreira, 2000; Kempen \& Hoenkamp, 1987; Levelt, 1989). Our data show, for the first time, that a similar process underlies pre-planning of noun phrases with subordination. This is important because, unlike verb-argument structures, complex noun phrases do not have verbal heads. We propose that similar to verb-arguments, planning scope for noun phrases is determined by semantic relations in the utterance to be produced, if these are important to the meaning (i.e. reference) of the utterance. Importantly this shows that non-linear message planning is not unique to verb-argument structures but can be found for complex noun phrases as well.

For verb-argument structures non-linear planning depends on subcategorisation of 
the verb (Bunger et al., 2013; Konopka \& Kuchinsky, 2015; Momma \& Ferreira, 2019) but the equivalent mechanisms for noun phrases is unknown. We propose that for noun phrases coordinating element (and, or) (Heim, 1982), prepositions and the possessive marker ('s) play an equivalent role (Barker, 1995, 2012; Kratzer, 2002). Indeed there is evidence that the preposition type, in particular the level of semantic integration indicated by the preposition (e.g. of, with), affects pre-planning of subordinated noun phrases (Solomon \& Pearlmutter, 2004). The need for unique idenfitiability of the referent, as proposed earlier, might trigger a Focus feature, as proposed by Jackendoff (1972), that is assigned to the referentially contrasting noun leading to a wide scope. In contrast to verbs, this feature is not necessarily morphologically realised. ${ }^{3}$

Non-linear planning for verb-argument structures has been taken as evidence that pre-planning on the conceptual or semantic level has a clause-level scope (see Bock \& Ferreira, 2014, Konopka \& Brown-Schmidt, 2014 for recent reviews). The idea is that semantic planning involves abstract - proposition-sized - scaffolds of the verb and its thematic arguments. It is not clear, however, how much semantic detail is involve for pre-planning verb-arguments. In other words, we do not know the structural depth of the planning process; arguments might be planned with or without embedded nouns (or attributes) (e.g. Brown-Schmidt \& Konopka, 2008). Further, the depth to which each argument is planned might depend not only on its linear position but also on its syntactic function (Do, 2018; Do \& Kaiser, 2019; Momma et al., 2015). Clausal planning might follow an agent-verb-patient strategy in which the argument structure relies on the semantic properties of the verb (i.e. the predicate) while there is no additional planning for noun phrases with embedded modifiers. Complex noun phrases might play a secondary role in pre-planning, subsequent to verb-argument structures. This possibility is an interesting

\footnotetext{
${ }^{3}$ In English, contrast can be marked prosodically (Selkirk, 1995) or using particles such as only, even and too that indicate contrastivity of a linguistic element with contextually available alternatives within the particle's scope (Paterson, Liversedge, Rowland \& Filik, 2003; Rooth, 1992).
} 
direction for future research; the question raised is whether pre-planning involves as much information as required by a higher level of representation or as much information as minimally required to release the production onset.

Returning to the central problem of this research: some process needs to decide the size of the sentence-initial chunk. This process may use relational factors such as agenthood or non-relational factors such as animacy or salience. The semantic plan that was determined at that stage might, however, not cascade into syntactic processing. The possibility that syntactic planning may be postponed until after production onset is in line with lexicalist theories of language production (e.g. Bock \& Levelt, 1994; F. Ferreira, 2000) but contradicts syntax-based accounts (e.g. Bock \& Ferreira, 2014; Chang et al., 2006; Levelt, 1989) to the extent that the syntactic structure is not required to guide advance grammatical encoding beyond the creation of a rudimentary (thematic) representation of the sentence. While some syntactic representation of the sentence structure might be anticipated, the syntactic organisation of the first noun phrase does not require grammatical encoding prior to production onset and may unfold as an emergent property of the production process. Alternatively, Konopka and Meyer (2014) proposed a horse-race process in which the use of either route depends on its prior activation. In situations when syntactic structure was pre-planed, a conceptually relational route must first have been activated. If the latter feeds into grammatical encoding, it adds to processing costs. What is not clear is whether, and if so under what conditions, the semantic plan cascades into syntactic encoding prior to production onset. This is an avenue for future research.

\section{Conclusion}

The experiments that we report in this paper provide evidence that grammatical encoding of syntactically complex noun phrases is grounded in the generation of semantic relations between message elements. Planning of syntactic relationships between phrase elements relies on the generation of a semantic structure which may influence advance 
planning beyond the first increment. Our results show that this property of the language production system, established for abstract representations of the verb and its argument(s), reproduces in modified noun phrases. Our findings suggest that it is not "the transition from thought to language" (E.-K. Lee et al., 2013) that underlies hierarchical dependencies but the relational organisation of the semantic representation itself.

\section{Acknowledgements}

This research was funded by XXX Scholarship scheme awarded to the first author. 


\section{References}

Allum, P. H. \& Wheeldon, L. R. (2007). Planning scope in spoken sentence production: The role of grammatical units. Journal of Experimental Psychology: Learning, Memory, and Cognition, 33(4), 791-810.

Allum, P. H. \& Wheeldon, L. R. (2009). Scope of lexical access in spoken sentence production: Implications for the conceptual-syntactic interface. Journal of Experimental Psychology: Learning, Memory, and Cognition, 35(5), 1240-1255.

Antón-Méndez, I. (2020). The role of verbs in sentence production. Frontiers in Psychology, 11.

Baayen, R. H., Davidson, D. J. \& Bates, D. M. (2008). Mixed-effects modeling with crossed random effects for subjects and items. Journal of Memory and Language, $59(4)$, $390-412$.

Baguley, T. (2012). Serious stats: A guide to advanced statistics for the behavioral sciences. Basingstoke: Palgrave Macmillan.

Barker, C. (1995). Possessive descriptions. Dissertations in Linguistics. Stanford: CSLI Publications.

Barker, C. (2012). Possessives and relational nouns. In K. von Heusinger, C. Maienborn \& P. Portner (Eds.), Semantics: An international handbook of natural language meaning (Vol. 2, pp. 1109-1130). Berlin: De Gruyter Mouton.

Barr, D. J. (2008). Analyzing 'visual world' eyetracking data using multilevel logistic regression. Journal of Memory and Language, 59(4), 457-474.

Barr, D. J., Levy, R., Scheepers, C. \& Tily, H. J. (2013). Random effects structure for confirmatory hypothesis testing: Keep it maximal. Journal of Memory and Language, $68(3), 255-278$.

Bates, D., Kliegl, R., Vasishth, S. \& Baayen, H. (2015). Parsimonious mixed models. arXiv preprint arXiv:1506.0496\%. 
Bates, E., D’Amico, S., Jacobsen, T., Székely, A., Andonova, E., Devescovi, A., ... Pléh, C. et al. (2003). Timed picture naming in seven languages. Psychonomic Bulletin \& Review, 10(2), 344-380.

Bock, J. K. \& Ferreira, V. S. (2014). Syntactically speaking. In M. Goldrick, V. S. Ferreira \& M. Miozzo (Eds.), The Oxford Handbook of Language Production (pp. 21-46). Oxford: Oxford University Press.

Bock, J. K. \& Griffin, Z. M. (2000). The persistence of structural priming: Transient activation or implicit learning? Journal of Experimental Psychology: General, 129(2), $177-192$.

Bock, J. K., Irwin, D. E. \& Davidson, D. J. (2004). Putting first things first. In J. M. Henderson \& F. Ferreira (Eds.), The interface of language, vision, and action: Eye movements and the visual world (pp. 249-278). New York, NY: Psychology Press.

Bock, J. K. \& Levelt, W. J. M. (1994). Language production: Grammatical encoding. In M. A. Gernsbacher (Ed.), Handbook of psycholinguistics (pp. 945-984). San Diego, CA: Academic Press.

Box, G. E. P. \& Cox, D. R. (1964). An analysis of transformations. Journal of the Royal Statistical Society, 26(2), 211-252.

Brown-Schmidt, S. \& Konopka, A. E. (2008). Little houses and casas pequeñas: Message formulation and syntactic form in unscripted speech with speakers of English and Spanish. Cognition, $109(2), 274-280$.

Brown-Schmidt, S. \& Konopka, A. E. (2015). Processes of incremental message planning during conversation. Psychonomic Bulletin \& Review, 22(3), 833-843.

Brown-Schmidt, S. \& Tanenhaus, M. K. (2006). Watching the eyes when talking about size: An investigation of message formulation and utterance planning. Journal of Memory and Language, 54(4), 592-609. 
Bunger, A., Papafragou, A. \& Trueswell, J. C. (2013). Event structure influences language production: Evidence from structural priming in motion event description. Journal of Memory and Language, 69(3), 299-323.

Carpenter, B., Gelman, A., Hoffman, M., Lee, D., Goodrich, B., Betancourt, M., ... Riddell, A. (2016). Stan: A probabilistic programming language. Journal of Statistical Software, 20.

Chang, F., Dell, G. S. \& Bock, J. K. (2006). Becoming syntactic. Psychological review, $113(2), 234-272$.

Clark, H. H. \& Fox Tree, J. E. (2002). Using uh and um in spontaneous speaking. Cognition, 84, 73-111.

Costa, A. \& Caramazza, A. (2002). The production of noun phrases in English and Spanish: Implications for the scope of phonological encoding in speech production. Journal of Memory and Language, 46(1), 178-198.

Dell, G. S. \& O'Seaghdha, P. G. (1992). Stages of lexical access in language production. Cognition, 42, 287-314.

Dickey, J. M., Lientz, B. P. et al. (1970). The weighted likelihood ratio, sharp hypotheses about chances, the order of a markov chain. The Annals of Mathematical Statistics, 41(1), 214-226.

Do, M. L. (2018). How to think before you speak: Getting from abstract thoughts to sentences (Doctoral dissertation, University of Southern California).

Do, M. L. \& Kaiser, E. (2019). Subjecthood and linear order in linguistic encoding: Evidence from the real-time production of wh-questions in English and Mandarin Chinese. Journal of Memory and Language, 105, 60-75.

Ferreira, F. (1991). Effects of length and syntactic complexity on initiation times for prepared utterances. Journal of Memory and Language, 30(2), 210-233. 
Ferreira, F. (2000). Syntax in language production: An approach using tree-adjoining grammars. In L. R. Wheeldon (Ed.), Aspects of language production (pp. 291-330). Hove, UK: Psychology Press.

Ferreira, F. \& Engelhardt, P. E. (2006). Syntax and production. In M. J. Traxler \& M. A. Gernsbacher (Eds.), Handbook of psycholinguistics (Vol. 2, pp. 61-91). Elsevier. doi:10.1016/B978-0-12-369374-7.X5000-7

Ferreira, F. \& Swets, B. (2002). How incremental is language production? evidence from the production of utterances requiring the computation of arithmetic sums. Journal of Memory and Language, 46(1), 57-84.

Ferreira, V. S. \& Slevc, L. R. (2007). Grammatical encoding. In M. G. Gaskell (Ed.), The Oxford Handbook of Psycholinguistics (pp. 453-470). Oxford, UK: Oxford University Press.

Fodor, J. A. (1983). The modularity of mind. Cambridge, MA: MIT press.

Garrett, M. F. (1975). Levels of processing in sentence production. In G. H. Bower (Ed.), The psychology of learning and motivation: Advances in research and theory (Vol. 9, pp. 133-177). New York: Academic Press.

Gelman, A., Carlin, J. B., Stern, H. S., Dunson, D. B., Vehtari, A. \& Rubin, D. B. (2014). Bayesian data analysis (3rd ed.). Chapman and Hall/CRC.

Gelman, A. \& Rubin, D. B. (1992). Inference from iterative simulation using multiple sequences. Statistical science, 7(4), 457-472.

Gibson, E. (2000). The dependency locality theory: A distance-based theory of linguistic complexity. In A. Marantz, Y. Miyashita \& W. O’Neil (Eds.), Image, language, brain (pp. 95-126). Cambridge, MA: MIT Press.

Gleitman, L. R., January, D., Nappa, R. \& Trueswell, J. C. (2007). On the give and take between event apprehension and utterance formulation. Journal of Memory and Language, 57(4), 544-569. 
Goodrich, B., Gabry, J., Ali, I. \& Brilleman, S. (2020). Rstanarm: Bayesian applied regression modeling via Stan. R package version 2.19.3. Retrieved from https://mc-stan.org/rstanarm

Griffin, Z. M. (2001). Gaze durations during speech reflect word selection and phonological encoding. Cognition, 82(1), B1-B14.

Griffin, Z. M. (2003). A reversed word length effect in coordinating the preparation and articulation of words in speaking. Psychonomic Bulletin \&3 Review, 10(3), 603-609.

Griffin, Z. M. (2004). Why look? Reasons for eye movements related to language production. In J. M. Henderson \& F. Ferreira (Eds.), The interface of language, vision, and action: Eye movements and the visual world (pp. 213-247). New York, NY: Psychology Press.

Griffin, Z. M. \& Bock, J. K. (2000). What the eyes say about speaking. Psychological Science, 11(4), 274-279.

Hardy, S. M., Segaert, K. \& Wheeldon, L. (2018). Healthy ageing and sentence production: Impaired lexical access in the context of intact syntactic planning. bioRxiv.

Hardy, S. M., Segaert, K. \& Wheeldon, L. (2019). Age-related disruption in the use of lexical information during sentence production, despite preserved syntactic planning.

Hardy, S. M., Segaert, K. \& Wheeldon, L. (2020). Healthy aging and sentence production: Disrupted lexical access in the context of intact syntactic planning. Frontiers in Psychology, 11, 257.

Heim, I. (1982). The semantics of definite and indefinite noun phrases (Doctoral dissertation, University of Massachusetts, Amherst).

Hoffman, M. D. \& Gelman, A. (2014). The No-U-Turn sampler: Adaptively setting path lengths in Hamiltonian Monte Carlo. Journal of Machine Learning Research, 15(1), $1593-1623$.

Jackendoff, R. S. (1972). Semantic interpretation in generative grammar. Cambridge, MA: MIT Press. 
Jeffreys, H. (1961). The theory of probability. Oxford: Oxford University Press, Clarendon Press.

Kempen, G. \& Hoenkamp, E. (1987). An incremental procedural grammar for sentence formulation. Cognitive science, 11(2), 201-258.

Konopka, A. E. (2012). Planning ahead: How recent experience with structures and words changes the scope of linguistic planning. Journal of Memory and Language, 66, $143-162$.

Konopka, A. E. \& Bock, J. K. (2009). Lexical or syntactic control of sentence formulation? Structural generalizations from idiom production. Cognitive Psychology, 58(1), 68-101.

Konopka, A. E. \& Brown-Schmidt, S. (2014). Message encoding. In M. Goldrick, V. S.

Ferreira \& M. Miozzo (Eds.), The Oxford Handbook of Language Production (pp. 3-20). Oxford: Oxford University Press.

Konopka, A. E. \& Kuchinsky, S. E. (2015). How message similarity shapes the timecourse of sentence formulation. Journal of Memory and Language, 84, 1-23.

Konopka, A. E. \& Meyer, A. S. (2014). Priming sentence planning. Cognitive Psychology, $73,1-40$.

Kratzer, A. (2002). The event argument and the semantics of verbs. Amherst: University of Massachusetts.

Kruschke, J. K. (2014). Doing bayesian data analysis: A tutorial with R, JAGS, and Stan (2nd ed.). Academic Press.

Kuchinsky, S. E. (2009). From seeing to saying: Perceiving, planning, producing (Doctoral dissertation, University of Illinois, Urbana-Champaign).

Kuchinsky, S. E., Bock, J. K. \& Irwin, D. E. (2011). Reversing the hands of time: Changing the mapping from seeing to saying. Journal of Experimental Psychology: Learning, Memory, and Cognition, 37(3), 748-756. 
Lee, E.-K., Brown-Schmidt, S. \& Watson, D. G. (2013). Ways of looking ahead: Hierarchical planning in language production. Cognition, 129(3), 544-562.

Lee, M. D. \& Wagenmakers, E.-J. (2014). Bayesian cognitive modeling: A practical course. Cambridge University Press.

Levelt, W. J. M. (1989). Speaking: From intention to articulation. MIT press.

Levelt, W. J. M. \& Maasen, B. (1981). Lexical search and order of mention in sentence production. In W. Kleine \& W. J. M. Levelt (Eds.), Crossing the boundaries in linguistics (pp. 221-252). Dordrecht: Reidel.

Levelt, W. J. M. \& Meyer, A. S. (2000). Word for word: Multiple lexical access in speech production. European Journal of Cognitive Psychology, 12(4), 433-452.

Lewis, R. L. \& Vasishth, S. (2005). An activation-based model of sentence processing as skilled memory retrieval. Cognitive Science, 29(3), 375-419.

Martin, R. C., Crowther, J. E., Knight, M., Tamborello II, F. P. \& Yang, C.-L. (2010). Planning in sentence production: Evidence for the phrase as a default planning scope. Cognition, 116(2), 177-192.

Martin, R. C., Yan, H. \& Schnur, T. T. (2014). Working memory and planning during sentence production. Acta Psychologica, 152, 120-132.

McElreath, R. (2016). Statistical rethinking: A bayesian course with examples in $R$ and Stan. CRC Press.

Meyer, A. S. (1996). Lexical access in phrase and sentence production: Results from picture-word interference experiments. Journal of Memory and Language, 35(4), 477-496.

Meyer, A. S. (1997). Conceptual influences on grammatical planning units. Language and Cognitive Processes, 12(5-6), 859-864.

Meyer, A. S. \& Lethaus, F. (2004). The use of eye tracking in studies of sentence generation. In J. M. Henderson \& F. Ferreira (Eds.), The interface of language, 
vision, and action: Eye movements and the visual world (pp. 191-211). New York, NY: Psychology Press.

Mirman, D., Dixon, J. A. \& Magnuson, J. S. (2008). Statistical and computational models of the visual world paradigm: Growth curves and individual differences. Journal of Memory and Language, 59(4), 475-494.

Momma, S. \& Ferreira, V. S. (2019). Beyond linear order: The role of argument structure in speaking. Cognitive Psychology, 114, 101228.

Momma, S., Slevc, L. R. \& Phillips, C. (2015). The timing of verb selection in japanese sentence production. Journal of Experimental Psychology: Learning, Memory, and Cognition, 42(5), 813-824.

Morey, R. D., Hoekstra, R., Rouder, J. N., Lee, M. D. \& Wagenmakers, E.-J. (2016). The fallacy of placing confidence in confidence intervals. Psychonomic Bulletin \& Review, 23(1), 103-123.

Myachykov, A., Garrod, S. \& Scheepers, C. (2012). Determinants of structural choice in visually situated sentence production. Acta Psychologica, $141(3)$, 304-315.

Nicenboim, B. \& Vasishth, S. (2016). Statistical methods for linguistic research: Foundational ideas - Part II. arXiv preprint arXiv:1602.00245.

Nottbusch, G. (2010). Grammatical planning, execution, and control in written sentence production. Reading and Writing, 23(7), 777-801.

Nottbusch, G., Weingarten, R. \& Sahel, S. (2007). From written word to written sentence production. In M. Torrance, L. van Waes \& D. W. W. Galbraith (Eds.), Writing and cognition: Research and applications (Vol. 20, pp. 31-53). Studies in writing. Amsterdam: Elsevier.

Paterson, K. B., Liversedge, S. P., Rowland, C. \& Filik, R. (2003). Children's comprehension of sentences with focus particles. Cognition, 89(3), 263-294.

Roeser, J. (2017). Planning scope in spoken and written sentence production (Doctoral dissertation, Nottingham Trent University). 
Roeser, J., Torrance, M. \& Baguley, T. (2019). Advance planning in written and spoken sentence production. Journal of Experimental Psychology: Learning, Memory, and Cognition, 45(11), 1983-2009. doi:10.1037/xlm0000685

Rooth, M. (1992). A theory of focus interpretation. Natural language semantics, 1(1), $75-116$.

Schriefers, H. \& Teruel, E. (1999). The production of noun phrases: A cross-linguistic comparison of French and German. In M. Hahn \& S. C. Stoness (Eds.), Proceedings of the 21st annual conference of the cognitive science society (pp. 637-642). Mahwah, NJ: Lawrence Erlbaum.

Schriefers, H., Teruel, E. \& Meinshausen, R. M. (1998). Producing simple sentences: Results from picture-word interference experiments. Journal of Memory and Language, 39(4), 609-632.

Selkirk, E. O. (1995). Sentence prosody: Intonation, stress, and phrasing. In J. Godsmith (Ed.), The handbook of phonological theory (Vol. 1, pp. 550-569). London: Blackwell.

Smith, M. \& Wheeldon, L. R. (1999). High level processing scope in spoken sentence production. Cognition, 73, 205-246.

Solomon, E. S. \& Pearlmutter, N. J. (2004). Semantic integration and syntactic planning in language production. Cognitive Psychology, 49(1), 1-46.

Sorensen, T., Hohenstein, S. \& Vasishth, S. (2016). Bayesian linear mixed models using stan: A tutorial for psychologists, linguists, and cognitive scientists. Quantitative Methods for Psychology, 12(3), 175-200.

Stan Development Team. (2015a). Stan: A C++ library for probability and sampling. http://mc-stan.org/. R package version 2.8.0.

Stan Development Team. (2015b). Stan modeling language user's guide and reference manual. http://mc-stan.org/. R package version 2.6.1.

Swets, B., Jacovina, M. E. \& Gerrig, R. J. (2013). Effects of conversational pressures on speech planning. Discourse Processes, 50(1), 23-51. 
Swets, B., Jacovina, M. E. \& Gerrig, R. J. (2014). Individual differences in the scope of speech planning: Evidence from eye-movements. Language and Cognition, 6(1), $12-44$.

Székely, A., D’Amico, S., Devescovi, A., Federmeier, K., Herron, D., Iyer, G., .. Bates, E. (2003). Timed picture naming: Extended norms and validation against previous studies. Behavior Research Methods, Instruments, \&6 Computers, 35(4), 621-633.

Székely, A., D’Amico, S., Devescovi, A., Federmeier, K., Herron, D., Iyer, G., .. Bates, E. (2005). Timed action and object naming. Cortex, 41(1), 7-25.

Székely, A., Jacobsen, T., D’Amico, S., Devescovi, A., Andonova, E., Herron, D., ... Wicha, N. et al. (2004). A new on-line resource for psycholinguistic studies. Journal of Memory and Language, 51(2), 247-250.

Tomlin, R. S. (1995). Focal attention, voice, and word order. In P. Downing \& M. Noonan (Eds.), Word order in discourse (pp. 517-552). Amsterdam: John Benjamins.

Van de Velde, M., Meyer, A. S. \& Konopka, A. E. (2014). Message formulation and structural assembly: Describing "easy" and "hard" events with preferred and dispreferred syntactic structures. Journal of Memory and Language, 71(1), 124-144.

Van Der Wege, M. M. (2009). Lexical entrainment and lexical differentiation in reference phrase choice. Journal of Memory and Language, 60(4), 448-463.

Wagenmakers, E.-J., Lodewyckx, T., Kuriyal, H. \& Grasman, R. (2010). Bayesian hypothesis testing for psychologists: A tutorial on the Savage-Dickey method. Cognitive psychology, 60(3), 158-189.

Wagner, V., Jescheniak, J. D. \& Schriefers, H. (2010). On the flexibility of grammatical advance planning during sentence production: Effects of cognitive load on multiple lexical access. Journal of Experimental Psychology: Learning, Memory, and Cognition, 36(2), 423-440.

Wheeldon, L. R. (2011). Generating spoken sentences: The relationship between words and syntax. Language and Linguistics Compass, 5/6, 310-321. 
Wheeldon, L. R. (2012). Producing spoken sentences: The scope of incremental planning. In S. Fuchs, M. Weirich, D. Pape \& P. Perrier (Eds.), Speech planning and dynamics (Vol. 1, pp. 97-118). Speech production and perception. Frankfurt, Germany: Peter Lang.

Wheeldon, L. R., Ohlson, N., Ashby, A. \& Gator, S. (2013). Lexical availability and grammatical encoding scope during spoken sentence production. The Quarterly Journal of Experimental Psychology, 66 (8), 1653-1673.

Wheeldon, L. R., Smith, M. C. \& Apperly, I. A. (2011). Repeating words in sentences: Effects of sentence structure. Journal of Experimental Psychology: Learning, Memory, and Cognition, 37(5), 1051-1064.

Zhao, L.-M., Alario, F.-X. \& Yang, Y.-F. (2015). Grammatical planning scope in sentence production: Further evidence for the functional phrase hypothesis. Applied Psycholinguistics, 36, 1059-1075.

Zhao, L.-M. \& Yang, Y.-F. (2013). Grammatical planning scope in sentence production: Evidence from Chinese sentences. Acta Psychologica Sinica, 45, 599-613.

Zhao, L.-M. \& Yang, Y.-F. (2016). Lexical planning in sentence production is highly incremental: Evidence from ERPs. PloS one, 11(1), 1-20. 


\section{Appendix A}

Sets of stimulus images (Experiment 1)

\begin{tabular}{|c|c|c|c|c|c|c|}
\hline Item & Scope & Target 1 & Target 2 & Comparator 1 & Comparator 2 & Colour \\
\hline 1 & N1 & obj131donkey & obj431sun & obj093chicken & obj431sun & red \\
\hline 1 & $\mathrm{~N} 2$ & obj131donkey & obj431sun & obj131donkey & obj434swing & red \\
\hline 2 & N1 & obj334porcupine & obj332popcorn & obj038bee & obj332popcorn & red \\
\hline 2 & $\mathrm{~N} 2$ & obj334porcupine & obj332popcorn & obj334porcupine & obj319pillow & red \\
\hline 3 & N1 & obj432swan & obj390shell & obj233ladybug & obj390shell & red \\
\hline 3 & $\mathrm{~N} 2$ & obj432swan & obj390shell & obj432swan & obj399skeleton & red \\
\hline 4 & N1 & obj249lizard & obj490bricks & obj480unicorn & obj490bricks & red \\
\hline 4 & $\mathrm{~N} 2$ & obj249lizard & obj490bricks & obj249lizard & obj492walnut & red \\
\hline 5 & N1 & obj157fish & obj095church & obj145elephant & obj095church & red \\
\hline 5 & $\mathrm{~N} 2$ & obj157fish & obj095church & obj157fish & obj114crackers & red \\
\hline 6 & N1 & obj402skunk & obj060bread & obj004alligator & obj060bread & red \\
\hline 6 & $\mathrm{~N} 2$ & obj402skunk & obj060bread & obj402skunk & obj062bridge & red \\
\hline 7 & N1 & obj313penguin & obj437table & obj170fox & obj437table & red \\
\hline 7 & $\mathrm{~N} 2$ & obj313penguin & obj437table & obj313penguin & obj438tail & red \\
\hline 8 & N1 & obj045bird & obj340purse & obj239leopard & obj340purse & red \\
\hline 8 & $\mathrm{~N} 2$ & obj045bird & obj340purse & obj045bird & obj337present & red \\
\hline
\end{tabular}


(continued)

\begin{tabular}{|c|c|c|c|c|c|c|}
\hline Item & Scope & Target 1 & Target 2 & Comparator 1 & Comparator 2 & Colour \\
\hline 9 & N1 & obj246lion & obj393shoe & obj416spider & obj393shoe & blue \\
\hline 9 & $\mathrm{~N} 2$ & obj246lion & obj393shoe & obj246lion & obj403sled & blue \\
\hline 10 & N1 & obj516worm & obj327plate & obj266monkey & obj327plate & blue \\
\hline 10 & $\mathrm{~N} 2$ & obj516worm & obj327plate & obj516worm & obj314piano & blue \\
\hline 11 & N1 & obj519zebra & obj367rug & obj297panda & obj367rug & blue \\
\hline 11 & $\mathrm{~N} 2$ & obj519zebra & obj367rug & obj519zebra & obj380scissors & blue \\
\hline 12 & N1 & obj343rabbit & obj181glasses & obj036beaver & obj181glasses & blue \\
\hline 12 & $\mathrm{~N} 2$ & obj343rabbit & obj181glasses & obj343rabbit & obj182globe & blue \\
\hline 13 & N1 & obj409snake & obj345radio & obj290owl & obj345radio & blue \\
\hline 13 & $\mathrm{~N} 2$ & obj409snake & obj345radio & obj409snake & obj350razor & blue \\
\hline 14 & N1 & obj419squirrel & obj196handcuffs & obj034bear & obj196handcuffs & blue \\
\hline 14 & $\mathrm{~N} 2$ & obj419squirrel & obj196handcuffs & obj419squirrel & obj193hammer & blue \\
\hline 15 & N1 & obj286octopus & obj472truck & obj171frog & obj472truck & blue \\
\hline 15 & $\mathrm{~N} 2$ & obj286octopus & obj472truck & obj286octopus & obj468trashcan & blue \\
\hline 16 & N1 & obj388shark & obj080hat & obj207hippo & obj080hat & blue \\
\hline 16 & $\mathrm{~N} 2$ & obj388shark & obj080hat & obj388shark & obj083carrot & blue \\
\hline
\end{tabular}


(continued)

\begin{tabular}{|c|c|c|c|c|c|c|}
\hline Item & Scope & Target 1 & Target 2 & Comparator 1 & Comparator 2 & Colour \\
\hline 17 & N1 & obj111cow & obj460grave & obj086cat & obj460grave & green \\
\hline 17 & $\mathrm{~N} 2$ & obj111cow & obj460grave & obj111cow & obj455tire & green \\
\hline 18 & N1 & obj476turtle & obj063broom & obj302parrot & obj063broom & green \\
\hline 18 & $\mathrm{~N} 2$ & obj476turtle & obj063broom & obj476turtle & obj064brush & green \\
\hline 19 & N1 & obj184goat & obj243lighthouse & obj178giraffe & obj243lighthouse & green \\
\hline 19 & $\mathrm{~N} 2$ & obj184goat & obj243lighthouse & obj184goat & obj236leaf & green \\
\hline 20 & N1 & obj067butterfly & obj108cork & obj501whale & obj108cork & green \\
\hline 20 & $\mathrm{~N} 2$ & obj067butterfly & obj108cork & obj067butterfly & obj132door & green \\
\hline 21 & N1 & obj126dinosaur & obj376saw & obj128dog & obj376saw & green \\
\hline 21 & $\mathrm{~N} 2$ & obj126dinosaur & obj376saw & obj126dinosaur & obj397sink & green \\
\hline 22 & N1 & obj268moose & obj417thread & obj475turkey & obj417thread & green \\
\hline 22 & $\mathrm{~N} 2$ & obj268moose & obj417thread & obj268moose & obj426stove & green \\
\hline 23 & N1 & obj121deer & obj259match & obj273mouse & obj259match & green \\
\hline 23 & $\mathrm{~N} 2$ & obj121deer & obj259match & obj121deer & obj260medal & green \\
\hline 24 & N1 & obj006ant & obj299paper & obj364rooster & obj299paper & green \\
\hline 24 & $\mathrm{~N} 2$ & obj006ant & obj299paper & obj006ant & obj298pants & green \\
\hline
\end{tabular}


(continued)

\begin{tabular}{|c|c|c|c|c|c|c|}
\hline Item & Scope & Target 1 & Target 2 & Comparator 1 & Comparator 2 & Colour \\
\hline 25 & N1 & obj072camel & obj465tractor & obj408snail & obj465tractor & yellow \\
\hline 25 & $\mathrm{~N} 2$ & obj072camel & obj465tractor & obj072camel & obj458toilet & yellow \\
\hline 26 & N1 & obj017baby & obj078canoe & obj284nurse & obj078canoe & yellow \\
\hline 26 & $\mathrm{~N} 2$ & obj017baby & obj078canoe & obj017baby & obj082carousel & yellow \\
\hline 27 & N1 & obj410snowman & obj129doll & obj122dentist & obj129doll & yellow \\
\hline 27 & $\mathrm{~N} 2$ & obj410snowman & obj129doll & obj410snowman & obj151feather & yellow \\
\hline 28 & N1 & obj112cowboy & obj192hamburger & obj357robot & obj192hamburger & yellow \\
\hline 28 & $\mathrm{~N} 2$ & obj112cowboy & obj192hamburger & obj112cowboy & obj191brush & yellow \\
\hline 29 & N1 & obj413soldier & obj235lawnmower & obj061bride & obj235lawnmower & yellow \\
\hline 29 & $\mathrm{~N} 2$ & obj413soldier & obj235lawnmower & obj413soldier & obj227kite & yellow \\
\hline 30 & N1 & obj338priest & obj369saddle & obj342queen & obj369saddle & yellow \\
\hline 30 & $\mathrm{~N} 2$ & obj338priest & obj369saddle & obj338priest & obj391boat & yellow \\
\hline 31 & N1 & obj513witch & obj149fan & obj489waiter & obj149fan & yellow \\
\hline 31 & $\mathrm{~N} 2$ & obj513witch & obj149fan & obj513witch & obj163flower & yellow \\
\hline 32 & N1 & obj127doctor & obj085castle & obj177ghost & obj085castle & yellow \\
\hline 32 & $\mathrm{~N} 2$ & obj127doctor & obj085castle & obj127doctor & obj092chest & yellow \\
\hline
\end{tabular}


Note: Each Scope pair was presented in both a prenominal and postnominal condition. Target 1, $2=$ referent of the first and second noun in the target phrase, Comparator $1,2=$ images presented along the target referents, Colour $=$ colour of target image. 


\section{Appendix B}

\section{Sets of stimulus images (Experiment 2)}

\begin{tabular}{|c|c|c|c|c|c|}
\hline Item & Scope & Target: modifier & Target: head & Prime: modifier & Prime: head \\
\hline 1 & head & obj006ant & obj213house & obj165fly & obj213house \\
\hline 1 & modifier & obj006ant & obj213house & obj165fly & obj167football \\
\hline 2 & head & obj246lion & obj217iron & obj385seal & obj217iron \\
\hline 2 & modifier & obj246lion & obj209hoof & obj385seal & obj217iron \\
\hline 3 & head & obj286octopus & obj064brush & obj501whale & obj064brush \\
\hline 3 & modifier & obj286octopus & obj064brush & obj501whale & obj307pear \\
\hline 4 & head & obj170fox & obj193hammer & obj171frog & obj193hammer \\
\hline 4 & modifier & obj170fox & obj396shower & obj171frog & obj193hammer \\
\hline 5 & head & obj184goat & obj295palmtree & obj249lizard & obj295palmtree \\
\hline 5 & modifier & obj184goat & obj295palmtree & obj249lizard & obj219jack \\
\hline 6 & head & obj034bear & obj395shovel & obj273mouse & obj395shovel \\
\hline 6 & modifier & obj034bear & obj244lightning & obj273mouse & obj395shovel \\
\hline 7 & head & obj086cat & obj189gun & obj384seahorse & obj189gun \\
\hline 7 & modifier & obj086cat & obj189gun & obj384seahorse & obj235lawnmower \\
\hline 8 & head & obj145elephant & obj050bone & obj266monkey & obj050bone \\
\hline 8 & modifier & obj145elephant & obj390shell & obj266monkey & obj050bone \\
\hline
\end{tabular}


(continued)

\begin{tabular}{|c|c|c|c|c|c|}
\hline Item & Scope & Target: modifier & Target: head & Prime: modifier & Prime: head \\
\hline 9 & head & obj133dragon & obj258mask & obj476turtle & obj258mask \\
\hline 9 & modifier & obj133dragon & obj258mask & obj476turtle & obj070cage \\
\hline 10 & head & obj130dolphin & obj282net & obj157fish & obj282net \\
\hline 10 & modifier & obj130dolphin & obj205highchair & obj157fish & obj282net \\
\hline 11 & head & obj251lobster & obj074can & obj313penguin & obj074can \\
\hline 11 & modifier & obj251lobster & obj074can & obj313penguin & obj437table \\
\hline 12 & head & obj111cow & obj115crib & obj493walrus & obj115crib \\
\hline 12 & modifier & obj111cow & obj263mirror & obj493walrus & obj115crib \\
\hline 13 & head & obj032bat & obj332popcorn & obj408snail & obj332popcorn \\
\hline 13 & modifier & obj032bat & obj332popcorn & obj408snail & obj150faucet \\
\hline 14 & head & obj067butterfly & obj198harp & obj072camel & obj198harp \\
\hline 14 & modifier & obj067butterfly & obj182globe & obj072camel & obj198harp \\
\hline 15 & head & obj480unicorn & obj060bread & obj519zebra & obj060bread \\
\hline 15 & modifier & obj480unicorn & obj060bread & obj519zebra & obj269mop \\
\hline 16 & head & obj126dinosaur & obj403sled & obj316pig & obj403sled \\
\hline 16 & modifier & obj126dinosaur & obj458toilet & obj316pig & obj403sled \\
\hline
\end{tabular}


(continued)

\begin{tabular}{|c|c|c|c|c|c|}
\hline Item & Scope & Target: modifier & Target: head & Prime: modifier & Prime: head \\
\hline 17 & head & obj344raccoon & obj318piggybank & obj516worm & obj318piggybank \\
\hline 17 & modifier & obj344raccoon & obj318piggybank & obj516worm & obj073camera \\
\hline 18 & head & obj121deer & obj183glove & obj178giraffe & obj183glove \\
\hline 18 & modifier & obj121deer & obj152fence & obj178giraffe & obj183glove \\
\hline 19 & head & obj113crab & obj149fan & obj402skunk & obj149fan \\
\hline 19 & modifier & obj113crab & obj149fan & obj402skunk & obj255mailbox \\
\hline 20 & head & obj128dog & obj363roof & obj388shark & obj363roof \\
\hline 20 & modifier & obj128dog & obj451thimble & obj388shark & obj363roof \\
\hline 21 & head & obj211horse & obj106comb & obj419squirrel & obj106comb \\
\hline 21 & modifier & obj211horse & obj106comb & obj419squirrel & obj079canopener \\
\hline 22 & head & obj416spider & obj163flower & obj475turkey & obj163flower \\
\hline 22 & modifier & obj416spider & obj124desk & obj475turkey & obj163flower \\
\hline 23 & head & obj122dentist & obj027bandaid & obj229knight & obj027bandaid \\
\hline 23 & modifier & obj122dentist & obj027bandaid & obj229knight & obj090cheese \\
\hline 24 & head & obj127doctor & obj042bench & obj357robot & obj042bench \\
\hline 24 & modifier & obj127doctor & obj315picture & obj357robot & obj042bench \\
\hline
\end{tabular}


(continued)

\begin{tabular}{|c|c|c|c|c|c|}
\hline Item & Scope & Target: modifier & Target: head & Prime: modifier & Prime: head \\
\hline 25 & head & obj179girl & obj227kite & obj513witch & obj227kite \\
\hline 25 & modifier & obj179girl & obj227kite & obj513witch & obj094chimney \\
\hline 26 & head & obj057boy & obj188guitar & obj147eskimo & obj188guitar \\
\hline 26 & modifier & obj057boy & obj062bridge & obj147eskimo & obj188guitar \\
\hline 27 & head & obj102clown & obj134drawer & obj323pirate & obj134drawer \\
\hline 27 & modifier & obj102clown & obj134drawer & obj323pirate & obj204helmet \\
\hline 28 & head & obj342queen & obj056box & obj489waiter & obj056box \\
\hline 28 & modifier & obj342queen & obj083carrot & obj489waiter & obj056box \\
\hline 29 & head & obj226king & obj254magnet & obj373sailor & obj254magnet \\
\hline 29 & modifier & obj226king & obj254magnet & obj373sailor & obj066butter \\
\hline 30 & head & obj061bride & obj356road & obj338priest & obj356road \\
\hline 30 & modifier & obj061bride & obj347rain & obj338priest & obj356road \\
\hline 31 & head & obj017baby & obj314piano & obj155fireman & obj314piano \\
\hline 31 & modifier & obj017baby & obj314piano & obj155fireman & obj368ruler \\
\hline 32 & head & obj284nurse & obj063broom & obj410snowman & obj063broom \\
\hline 32 & modifier & obj284nurse & obj181glasses & obj410snowman & obj063broom \\
\hline
\end{tabular}


Note: Each Contrast Noun pair was present in both a prenominal and postnominal condition. Scope on head is narrow scope in postnominals and wide scope in prenominals, and vice versa. Target $=$ referent of the head and the modifier noun in the target phrase, Prime = images presented along the target referents which constitute the prime targets). 


\section{Appendix C}

\section{Results: Prime trials (Experiment 2)}

This section summarises the results for prime trials. We present the results for the written session first and then present the results of the spoken session. Figure C1a shows the posterior parameter values for the time of gaze-shift from N1 to N2 in the written session. Evidence for scope effects was negligible $(\mathrm{BF}<0.06)$. Similarly, the probability of gaze shift to occur before production onset showed negligible evidence for a scope effect $(\mathrm{BF}<$ 0.6). Estimated probability was similar in the wide scope condition both for prenominal modifier phrases and postnominal modifier phrases (prenominal: narrow-scope $\hat{\mu}=.44$, 95\% PI $[.34, .54]$, wide-scope $\hat{\mu}=.52,95 \%$ PI $[.41, .62]$; postnominal: narrow-scope $\hat{\mu}=$ $.52,95 \%$ PI $[.42, .63]$, wide-scope $\hat{\mu}=.5,95 \%$ PI $[.4, .61])$. The inferred parameter values for the onset latency can be found in Figure C1b. Evidence for scope effects was negligible $(\mathrm{BF}<0.2)$. 


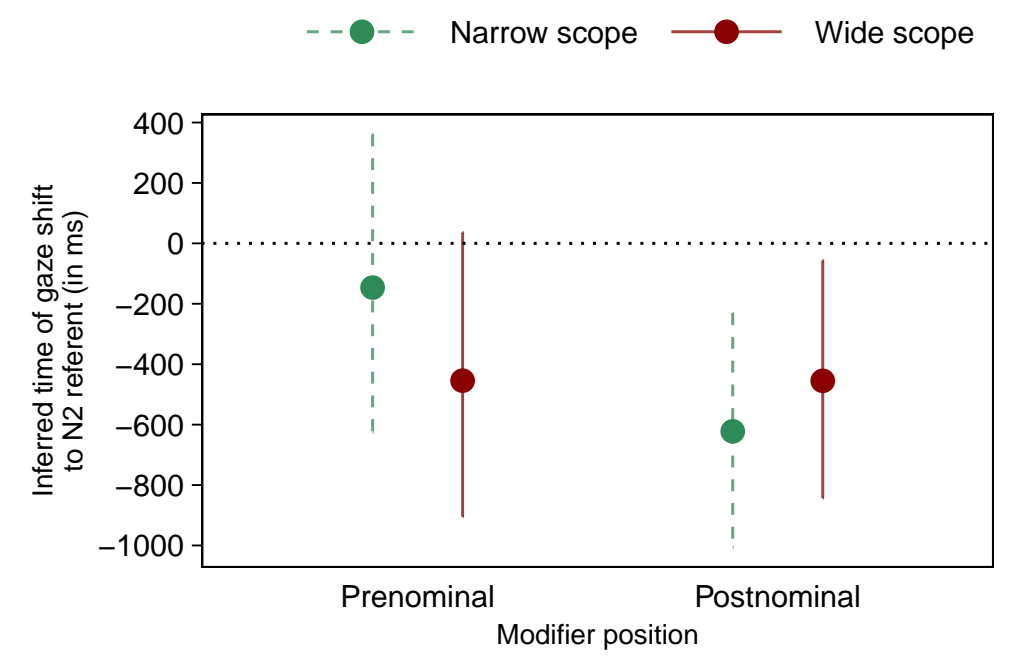

(a) Time of gaze shift from N1 to N2 referent. The dotted line

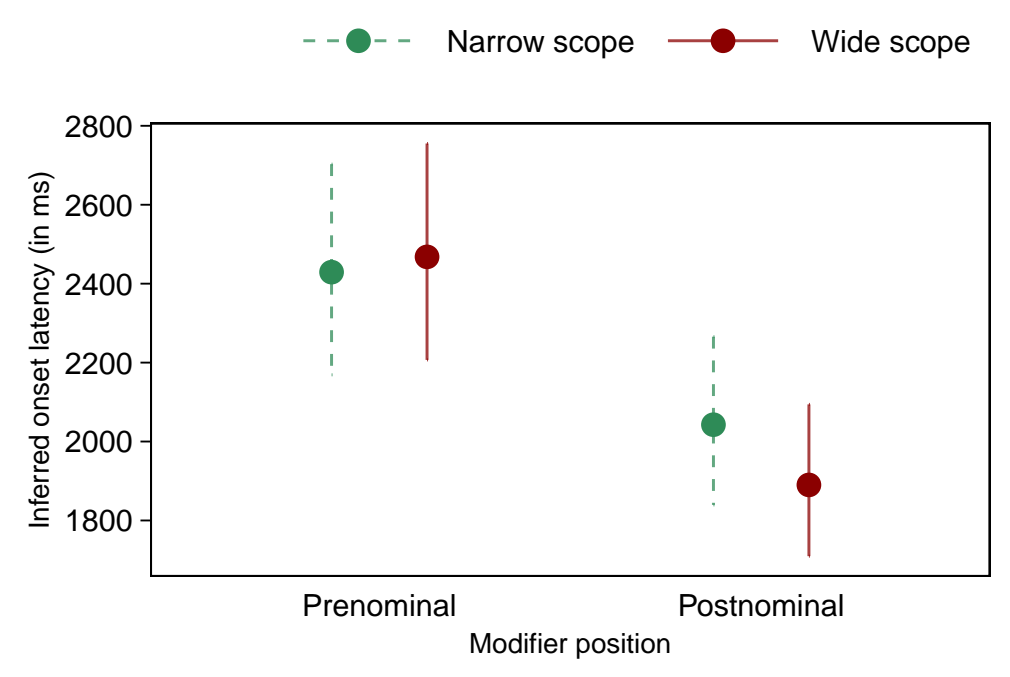

(b) Onset latency

indicates the production onset.

\section{Figure C1}

Experiment 2, Written production. Mean gaze shift and production onset latencies. Error bars represent 95\% PIs. Parameter estimates from full-factorial Modality $\times$ prime/target $\times$ Modifier-position $\times$ Scope Bayesian linear mixed effects models. 
For the spoken sessions we found the following results. Figure C2a shows the posterior inferred parameter values for the time of gaze-shift from N1 to N2 relative to the production onset. Evidence for scope effects was negligible $(\mathrm{BF}<0.08)$. Although we found negligible evidence for a scope effect in the gaze-shift timing data, we observed evidence for a larger probability for gaze shift to happen before production onset under wide scope in phrases with postnominal modification but not for phrases with prenominal modification (prenominal: narrow-scope $\hat{\mu}=.45,95 \%$ PI $[.33, .58]$, wide-scope $\hat{\mu}=.5,95 \%$ PI $[.36, .63], \mathrm{BF}<0.4$; postnominal: narrow-scope $\hat{\mu}=.65,95 \%$ PI $[.56, .74]$, wide-scope $\hat{\mu}$ $=.78,95 \% \mathrm{PI}[.71, .85], \mathrm{BF}=6.78)$. The inferred parameter values for the onset latency can be found in Figure C2b. Evidence for scope effects was negligible $(\mathrm{BF}<0.09)$. 


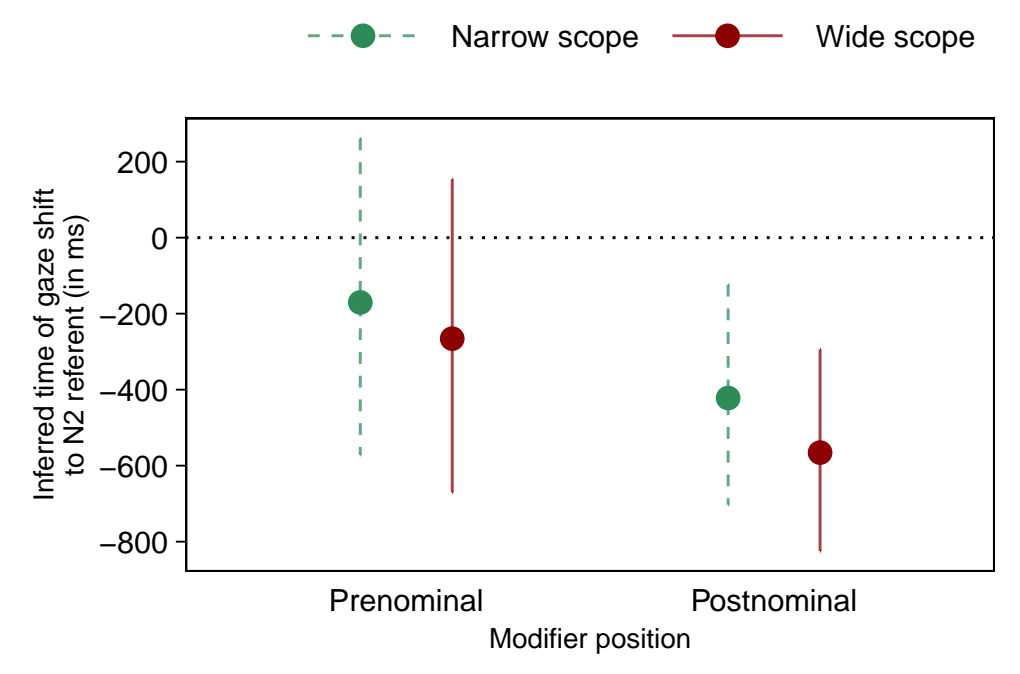

(a) Time of gaze shift from N1 to N2 referent. The dotted line

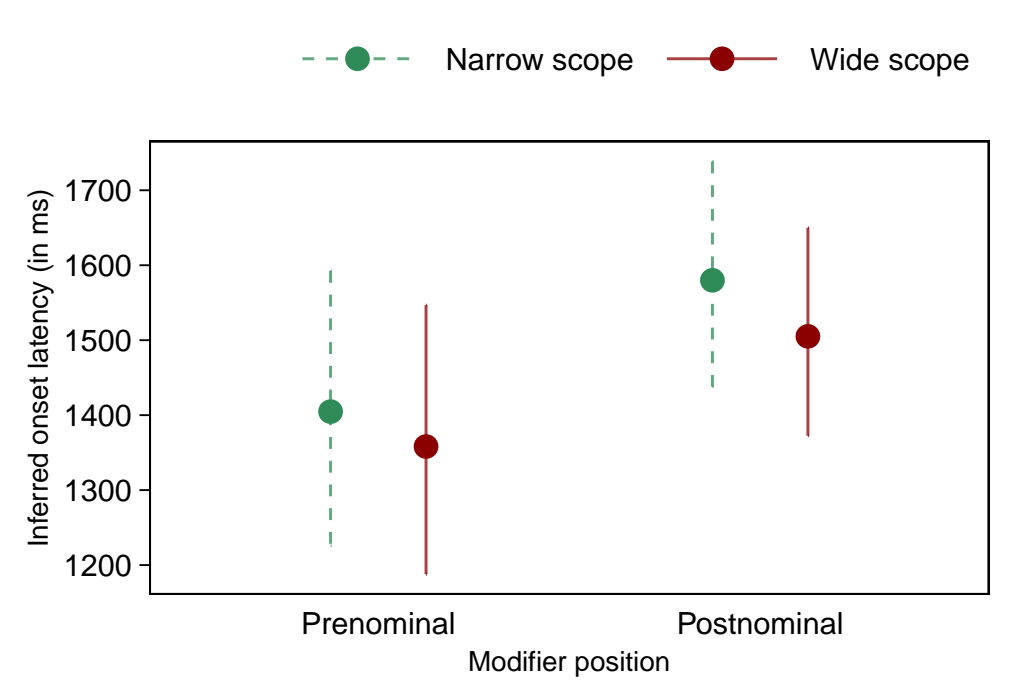

(b) Onset latency.

indicates the production onset.

\section{Figure C2}

Experiment 2, Spoken production. Mean gaze shift and production onset latencies. Error bars represent 95\% PIs. Parameter estimates from full-factorial Modality $\times$ prime/target $\times$ Modifier-position $\times$ Scope Bayesian linear mixed effects models. 